Ferdinand Mberamihigo, Gilles-Maurice de Schryver* et Koen Bostoen

\title{
Entre verbe et adverbe : Grammaticalisation et dégrammaticalisation du marqueur épistémique umeengo/umeenga en kirundi (bantou, JD62)
}

DOI 10.1515/jall-2016-0011

Résumé : La présente étude concerne l'évolution sémantique et formelle d'un adverbe épistémique du kirundi, umeengo/umeenga, les deux formes constituant des variantes libres. Elle est une des premières études s'inscrivant en linguistique de corpus pour les langues bantoues, une démarche rarement adoptée pour cette famille de langues, de même qu'elle étudie une catégorie grammaticale généralement négligée dans les études linguistiques, c'est-à-dire l'adverbe. Nous décrivons les différents emplois de umeengo/umeenga : comme adverbe, mais aussi comme comparatif, et dans les emplois du verbe qui en émerge à l'étape suivante dans son évolution. Ses origines sont éclairées par un indice retrouvé en kinyarwanda, langue proche du kirundi, ainsi que dans la variante dialectale du kirundi parlée à l'ouest du Burundi, dans la région de l'Imbo. D’après ces éléments, il apparaît que cet adverbe est formé par univerbation, plus précisément par fusion d'un verbe conjugué, umenya (du verbe -meny- " connaître ») et du quotatif ngo « que ». Dans la suite du processus, à partir de cet adverbe épistémique, se forme un nouveau verbe, -meeng- « croire ; penser », différent de celui du départ, tant dans le sens qu'au niveau de la forme. Une telle évolution au sein de l'adverbe, qui naît d'un verbe et débouche lui-même à la naissance d'un autre verbe, est rare dans les langues du monde, de là l'intérêt de notre analyse. Dans cette étude, nous nous interrogeons sur la nature des processus à l'œuvre. Nous montrons que les changements structurels et sémantiques par lesquels l'adverbe umeengo/umeenga s'est

\footnotetext{
*Corresponding author: Gilles-Maurice de Schryver, BantUGent - UGent Centre for Bantu Studies, Université de Gand (UGent), Belgique; Département de langues africaines, Université de Pretoria, Afrique du Sud, E-mail: gillesmaurice.deschryver@ugent.be Ferdinand Mberamihigo, Département de langues et littératures africaines, Université du Burundi, Burundi; BantUGent - UGent Centre for Bantu Studies, Université de Gand (UGent), Belgique, E-mail: fmberamihigo@gmail.com Koen Bostoen, BantUGent - UGent Centre for Bantu Studies, Université de Gand (UGent), Belgique, E-mail: koen.bostoen@ugent.be
} 
formé sont à mettre sur le compte de la grammaticalisation. Dans le même ordre d'idée, nous analysons la formation du verbe -meeng- à partir de l'adverbe comme le résultat d'une dégrammaticalisation.

Mots-clés : bantou, kirundi, modalité, possibilité, épistémique, adverbe, grammaticalisation, lexicalisation, dégrammaticalisation, délexicalisation

\section{Introduction ${ }^{1}$}

L'adverbe est généralement peu étudié par rapport à d'autres catégories grammaticales comme le nom et le verbe ; pourtant les mécanismes qui s'observent dans sa formation et son fonctionnement sont d'une grande richesse et contribuent à une meilleure compréhension des phénomènes linguistiques. Plus particulièrement l'étude de l'adverbe apporte davantage de lumière dans l'éclairage des processus de formation des mots et de changements sémantiques. Au sein des langues bantoues, peu d'études ont été consacrées à l'adverbe. On peut citer un certain nombre d'ouvrages sur le setswana, dont l'étude de Segopolo (1992), ainsi que l'article écrit par Le Roux (2007); l'analyse menée sur le luganda au sujet des adverbes de manière mérite également d'être évoqué (Mould 1977). Dans notre étude sur la modalité en kirundi, langue parlée au Burundi, nous apportons également une contribution à ce sujet en consacrant un chapitre aux adverbes épistémiques (Mberamihigo 2014). Il s'agit sans doute de la première étude systématique de ce genre d'adverbes pour une langue bantoue. Pour le kirundi, si nous pouvons faire préalablement un état des lieux du traitement de l'adverbe, nous constatons que la brièveté de la manière dont cette classe est abordée témoigne de ce moindre intérêt, ou de l'évitement dont il fait l'objet de manière générale. Elle n'est pas traitée dans la grammaire du kirundi de Meeussen (1959), pas plus qu'elle n'est évoquée dans l'index de cette étude. Ntahokaja (1994) réserve à l'adverbe un traitement dans le cadre des classes de mots. Néanmoins, il ne donne le détail que sur quatre espèces : les adverbes de lieu, les adverbes de temps, les adverbes de manière et les adverbes d'affirmation et de négation. Quant à Cristini (2001), il leur consacre un chapitre, où il en décrit la formation, en grandes lignes. Il répertorie les

1 Les recherches pour cet article ont été effectuées dans le cadre du doctorat du premier auteur (2010-2014) sous la direction des deux autres auteurs. La thèse a été faite en cotutelle entre l'Université libre de Bruxelles et l'Université de Gand et a été financée par une bourse du Gouvernement du Burundi. Nous tenons à remercier deux collègues anonymes ainsi que Sebastian Dom pour leurs commentaires constructifs sur une version antérieure de cet article. 
adverbes d'intensité, de manière, d'opinion, d'interrogation, de temps et de lieu (ou adverbes locatifs). Zorc \& Nibagwire (2007), pour leur part, dans leur étude comparative du kirundi et du kinyarwanda, incluent également un chapitre sur les adverbes, et répertorient six classes : adverbes de temps, de lieu, de manière, de degré ou intensité, de mesure (ou encore quantité ou fréquence), et enfin adverbes d'opinion. Dans cette dernière classe, ils citent ce qui correspond aux adverbes épistémiques, mais pour le kirundi seuls deux éléments y trouvent leur compte : un épistémique, kokó " really, truly », et ce qui est en réalité un évidentiel inférentiel, búrya, qu'ils traduisent par " in fact, essentially ». Le troisième élément censé être du kirundi d'après les auteurs, est buruúndu, correspondant au kinyarwanda buruundú " definitely, for sure, decidedly, for good ", les deux n'ayant qu'une différence de tonalité. Mais en réalité cet épistémique de certitude n'est utilisé par les locuteurs burundais que par influence du kinyarwanda. Notre corpus du kirundi n'en fait état dans l'usage qu'à partir de la décennie 1990s. Le propos des auteurs au sujet des adverbes est lui-même un signe de volatilité de cette catégorie : ils précisent, en effet, que, normalement, ces adverbes d'opinion sont, ou bien des interjections, ou bien des idéophones (Zorc \& Nibagwire 2007, p. 321), traduisant par là la difficulté à appréhender les éléments de la catégorie des adverbes avec des critères stables.

La délimitation de cette catégorie est en effet problématique; beaucoup de ceux qui ont eu à la traiter ont montré qu'elle n'est pas aussi nettement circonscrite que les autres catégories. Dans notre cadre opératoire, la définition des adverbes fournie par Hoye (1997 : 140) peut être prise comme référence, bien qu'elle-même ne les présente essentiellement que par ce qu'ils ne sont pas. Son apport est de détailler le rôle fonctionnel de l'adverbe : "Adverbs are a heterogeneous group of items which contrast with the other main word-classes, nouns, verbs and adjectives. They function in a variety of ways, modifying other elements in clause structure, such as adjectives, other adverbs or even the clause as a whole, but their most frequent role is to specify the mode of action of the verb ». Il nous faut préciser, d'emblée, que nous distinguons les adverbes et les adverbiaux, à l'instar de Ramat (2011 : 503). Ces derniers renvoient plutôt à des entités remplissant une fonction similaire à celle des adverbes. Il pourra notamment s'agir de locutions adverbiales.

C'est pour apporter une contribution en ce qui concerne la formation de l'adverbe, en particulier dans les langues bantoues, que nous proposons le présent article. Notre objectif est d'étudier l'émergence et le fonctionnement des formes umeengo/umeenga, deux variantes d'un adverbe modal du kirundi (JD62), langue bantoue de la zone interlacustre. Nous étudions son parcours, son émergence en tant qu'adverbe épistémique et son développement dans le domaine post-modal. Nous montrons de quelle manière il émerge par 
l'action des mécanismes simultanés de grammaticalisation et lexicalisation, et dans quelle mesure, dans son parcours post-modal, il subit à la fois la dégrammaticalisation et la délexicalisation, des phénomènes peu décrits en bantou. Nous en offrons une description détaillée basée sur un corpus de textes kirundi. D'une manière générale, la linguistique de corpus reste d'une exploitation limitée dans les langues bantoues (Kawalya et al. 2014). Il s'agit pourtant d'un domaine dont l'application débouche sur des conclusions concrètes dont le plus grand mérite est de partir des faits tirés de situations réelles de communication. De la sorte, pour autant que l'on utilise des données équilibrées, les conclusions peuvent être généralisées à toute la langue, car le corpus en est le représentant. Une description basée sur un corpus permet également de mener une approche statistique sur les phénomènes à l'étude. Le corpus que nous employons est d'une taille de deux million de tokens. Il s'agit d'un corpus brut qui comprend des 'textes' oraux et écrits situés entre 1940 et 2012, couvrant ainsi une période de huit décennies. En outre, les textes couvrent plusieurs genres/ sujets, d'un total de dix-sept. Par genre/sujet, nous entendons des catégories telles que les textes juridiques, les chansons, les romans, les textes en rapport avec l'éducation, la culture, l'histoire, etc. Le souci est d'avoir un corpus qui puisse regrouper des productions linguistiques recueillies sur un intervalle de temps suffisamment large et comportant une grande diversité thématique autant que faire se peut. Le tout est rassemblé sous forme de textes électroniques analysables par le logiciel WordSmith Tools, adapté à cette fin. Avec une telle approche, nous sommes assurés de pouvoir mener des analyses crédibles sur la fréquence et la distribution des marqueurs étudiés.

Nous étudions les adverbes dans le cadre de la modalité. Comme discuté par Nuyts (2005, 2006), cette dernière fait l'objet de beaucoup de définitions qui varient en fonction des regards des uns et des autres. Elle est parfois définie comme étant « l'expression de l'attitude du locuteur par rapport au contenu propositionnel de son énoncé » (Le Querler 1996 : 61) ou une catégorie « qui permet d'expliciter ce que sont les positions du sujet parlant par rapport à son interlocuteur » (Charaudeau 1992 : 572), et peut même être vue " in terms of a lack of factivity, or, from a different perspective, as the relativization of the validity of a proposition with respect to a certain background " (Narrog 2010 : 392). Nous pouvons résumer toutes ces définitions en présentant leur noyau commun, selon lequel la modalité sera définie comme le domaine sémantique couvrant l'expression du point ou de l'attitude du locuteur par rapport à la réalité d'un fait énoncé. La modalité épistémique à laquelle se rapporte l'adverbe que nous étudions est généralement définie de la façon suivante : " [...] concerns an indication of the estimation, [...] typically, but not necessarily by the speaker, of the chances that the state of affairs expressed in the clause applies in the world or 
not, or, in other words, of the degree of probability of the state of affairs " (Nuyts 2005 : 10). Dans la perspective de van der Auwera \& Plungian (1998) le domaine de la modalité épistémique peut également être divisé en possibilité épistémique, correspondant à une faible estimation de la vérité d'un fait, et la nécessité épistémique, se rapportant à une forte estimation de cette vérité, et même à la certitude absolue. Comme nous le montrons dans cet article, umeengo/umeenga marque la possibilité épistémique en kirundi. En même temps, il marque l'évidentialité, qui réfère à la source de la connaissance des faits décrits par le locuteur : "It involves a characterization of the origins of knowledge about the state of affairs, or of the compatibility of the (postulated) state of affairs with the general epistemological background of the issuer » (Nuyts 2006 : 10). Umeengo/umeenga se trouve à l'intersection entre la possibilité épistémique et l'évidentialité, une polysémie qui est courante dans les langues du monde (Aikhenvald 2004 : 6).

Notre article est subdivisé en six sections. Après la présente introduction, nous présentons à la Section 2 un bref aperçu des adverbes épistémiques du kirundi. La Section 3 est consacrée à la description de la variation formelle et du fonctionnement de l'adverbe umeengo/umeenga comme marque de la possibilité épistémique et de l'évidentialité. À la Section 4, nous reconstruisons l'émergence de cet adverbe par les processus de grammaticalisation et de lexicalisation. À la Section 5, nous décrivons ses emplois en tant que comparatif et suivons son développement vers l'émergence d'un verbe par les processus de dégrammaticalisation et délexicalisation. Une synthèse et les conclusions sont présentées à la Section 6.

\section{Les adverbes épistémiques du kirundi}

Le kirundi compte sept adverbes épistémiques dont six expriment la possibilité épistémique et se traduisent par " peut-être » en français : ngirango, umeengo/ umeenga, kuumburé, nkeeka, kurúubu, ubóna. Comme décrit en détail dans Mberamihigo (2014 : 275-335), ils n’ont pas exactement la même portée sémantique. Le septième, à savoir kokó, exprime la certitude ou la nécessité épistémique. Comme la Figure 1 montre, umeengo/umeenga est l'adverbe épistémique qui occupe le deuxième rang en termes de fréquence. Il représente 27\% de la totalité des adverbes épistémiques attestés dans notre corpus, ce qui correspond en termes absolus à 491 attestations.

Parmi les sept, umeengo/umeenga présente au moins deux particularités. D'une part, il est le seul à avoir une valeur évidentielle en plus de sa valeur épistémique. Comparons l'usage de ngirango en (1) avec celui d'umeenga en (2). 


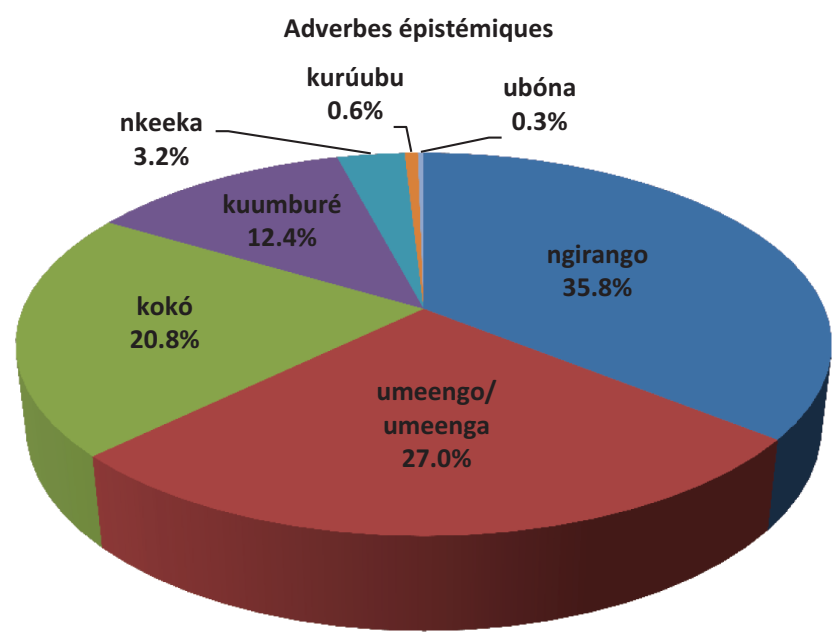

Figure 1: Fréquence des adverbes épistémiques en kirundi.

(1) Twebwe turi abarimu twigisha mw'ishule Kaminuza y’i Bujumbura ngirango muramaze kuhumva.

tweebwé tu-ri a-ba-áarimú tu-ígiish-a

nous $\quad \mathrm{PS}_{1 \mathrm{pl}}$-être $\mathrm{AUG}_{2}-\mathrm{PN}_{2}$-professeur $\quad \mathrm{PS}_{1 \mathrm{pl}}$-enseigner-IPFV

mu i- $\emptyset$-shuúre $\varnothing$-kaminúuza i Bujuumbura

$\mathrm{LOC}_{18} \quad \mathrm{AUG}_{5}-\mathrm{PN}_{5}$-école $\mathrm{PN}_{9}$-université $\mathrm{LOC}_{19}$ Bujumbura

ngirango mu-ra-mar-ye ku-ha-úmv-a

peut-etre $\mathrm{PS}_{2 \mathrm{pl}}$-DJ-finir-PFV $\mathrm{PN}_{15}-\mathrm{PO}_{16}$-entendre-VF

Nous, nous sommes des professeurs, nous enseignons à l'Université de Bujumbura, peut-être vous avez déjà entendu parler d'elle.

(Mpinga, Culture traditionnelle, 1980s)

(2) Babaye bacegera Igisengero, bumva umenga hari urwamo rwinshi... bá-á-bá-ye ba ${ }^{\mathrm{H}}$-ki-egeer-a i-ki-seengero

$\mathrm{PS}_{2}$-PE-être-PFV $\quad \mathrm{PS}_{2}$.CJC-PERST-s'approcher-IPFV $\quad \mathrm{AUG}_{7}$-PN $\mathrm{PN}_{7}$-temple

ba-úmv-a umeenga ha-ri u-ru-áamo

$\mathrm{PS}_{2}$-entendre-IPFV apparemment $\mathrm{PS}_{16}$-être $\mathrm{AUG}_{11}-\mathrm{PN}_{11}$-bruit

ru-ínshi

$\mathrm{PP}_{11}$-beaucoup

Ils s'approchaient encore du temple quand ils entendirent que, apparemment, il y avait beaucoup de bruit...

(Yaga, Religion, 1960s) 
Les professeurs d'université en (1) se présentent à leurs informateurs ruraux au cours d'une enquête et ne se fondent sur aucun indice particulier pour avancer que leurs interlocuteurs seraient peut-être au courant de l'existence de leur université. Par contre, en (2), les personnes s'approchant du temple se fondent sur un bruit dont l'intensité ne leur était pas tout à fait sûre. L'adverbe ne marque pas uniquement la possibilité épistémique ici, mais aussi l'évidentialité qui repose sur un indice sonore comme source. Un même marqueur peut véhiculer ces deux valeurs à la fois (van der Auwera \& Plungian 1998, Plungian 2001, Cornillie 2009). En (2), umeenga ne peut être remplacé par aucun autre des adverbes épistémiques. Par contre, ngirango en (1) est interchangeable avec les autres sauf umeengo/umeenga.

De surcroît, umeengo/umeenga se distingue par le fait qu'il s'est grammaticalisé non seulement à partir d'un verbe, comme les autres adverbes exprimant la possibilité épistémique, mais qu'il est lui-même à l'origine d'une nouvelle forme verbale. Celle-ci résulte, comme nous démontrons plus loin, d'un processus de dégrammaticalisation peu courant.

\section{Formes et usages synchroniques de l'adverbe umeengo/umeenga}

Dans cette section, nous décrivons la variabilité formelle (3.1) ainsi que la grande diversité d'emplois (3.2) de l'adverbe umeengo/umeenga.

\subsection{Un adverbe, deux variantes formelles}

Les formes umeengo et umeenga sous lesquelles l'adverbe en question se manifeste peuvent être considérées comme des variantes libres, puisque rien ne les distingue sur les plans sémantique et syntaxique. Les deux formes sont toujours interchangeables, quoi que soit le sens exact qu'elles expriment ou la position linéaire dans la proposition qu'elles occupent. Leur coexistence est d'ailleurs plus ancienne que notre corpus, car les deux formes sont attestées dès le début de la période couverte par notre corpus. Le Tableau 1 reproduit la distribution diachronique des deux variantes dans le corpus, tandis que la Figure 2 nous informe sur leurs tendances d'usage à travers le temps.

Pris dans sa globalité, notre corpus corrobore le constat de Rodegem (1970, p. 262) que la variante avec finale -o est la plus fréquente. Par contre, et ce qui 
Table 1: Distribution diachronique de umeengo et umeenga au sein de notre corpus : répartition par moyenne de 10.000 mots dans le corpus.

\begin{tabular}{rrrrrrrrr}
\hline & $1940 s$ & $1950 s$ & $1960 s$ & $1970 s$ & $1980 s$ & $1990 s$ & $2000 s$ & $2010 s$ \\
\hline umeengo & 2,0 & 7,5 & 1,4 & 4,4 & 1,7 & 2,6 & 1,4 & 1,8 \\
umeenga & 0,6 & 0,0 & 0,6 & 0,5 & 0,8 & 0,1 & 0,6 & 0,5 \\
\hline
\end{tabular}

Tendances d'usage de umeengo et umeenga à travers le temps

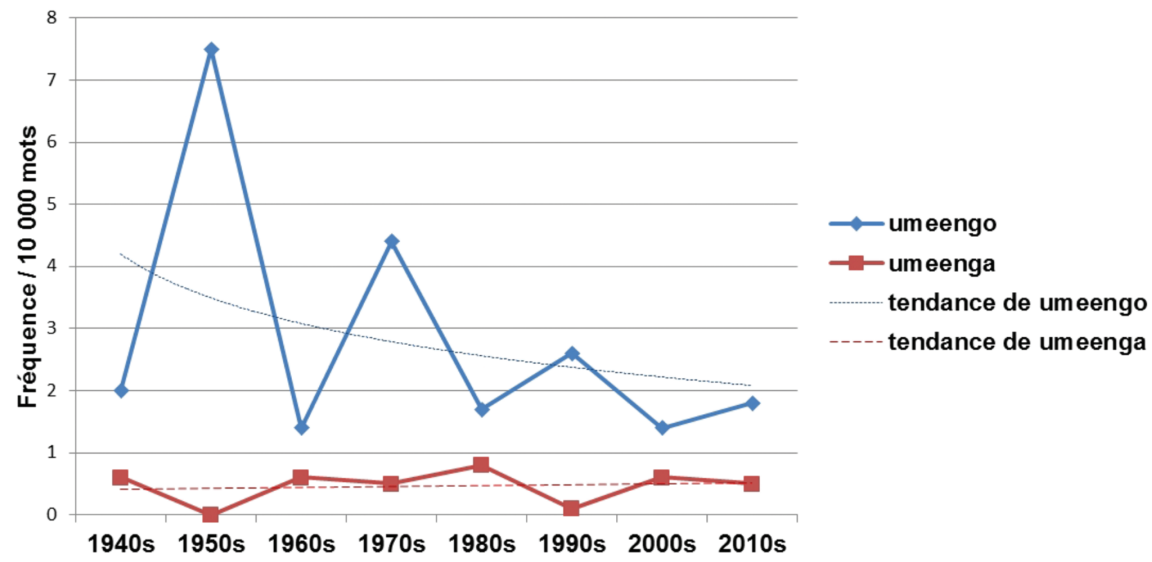

Figure 2: Tendances d'usage de umeengo et umeenga à travers le temps.

est révélé ici pour la première fois, c'est que la fréquence d'emploi de umeengo manifeste une tendance à décliner à travers le temps, de environ quatre occurrences pour chaque tranche de 10000 mots dans les années 1940s à environ deux occurrences dans les années 2010s, tandis que celle de umeenga reste relativement stable, avec une occurrence tous les 20000 mots. Autrement dit, bien que la distinction entre umeengo et umeenga soit plus ancienne que la période couverte par notre corpus, ce dernier indique que umeenga continue à être employée avec la même fréquence de départ, tandis que l'usage de umeengo est réduit de moitié en seulement huit décennies. La différence ne doit pas être cherchée au niveau de leur distribution auprès des locuteurs, étant donné que chaque décennie de notre corpus compte des données provenant de multiples sources bien différentes. Nous argumentons ci-dessous qu'on assiste ici au processus de dégrammaticalisation de l'adverbe umeengo. 


\subsection{Umeengo/umeenga comme adverbe épistémique évidentiel}

Les formes umeengo et umeenga peuvent être traduites en français par « probablement, apparemment, vraisemblablement ». Dans leur emploi actuel, les valeurs épistémique et évidentielle prédominent. En y recourant, un locuteur signale qu'il émet une assertion sans certitude absolue, d'où la valeur épistémique de l'adverbe. Dans la mesure où le locuteur se fonde sur un indice externe, l'adverbe peut être évidentiel en même temps. Toutefois, cet indice reste toujours insuffisant ou incomplet pour convaincre le locuteur d'être dans le vrai. Le chevauchement entre l'épistémique et l'évidentiel s'observe typiquement dans un évidentiel inférentiel, comme illustré en (3). L'évidentialité se fonde ici sur une expérience visuelle: la personne regarde et se rend compte de l'absence de son bien. La possibilité épistémique s'explique par le fait que son observation demeure incertaine.

(3) Aha nahabitse ikintu, none umengo baragikuyeho...

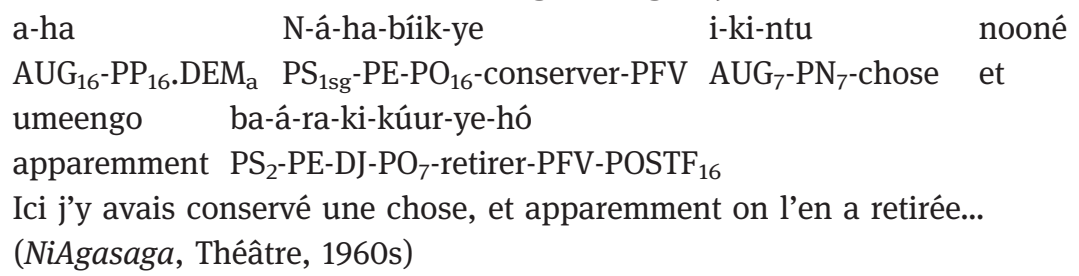

En (4), l'évaluation épistémique se base aussi sur la vue, qui n'est pas non plus assez nette pour obtenir une certitude absolue. Par conséquent, umeengo est ici plus proche de « apparemment » que de " peut-être ».

(4) Yinjiranye n'umukobwa, kandi umengo ni Janine!

a-ínjir-an-ye na u-mu-koóbwa kaándi
$\mathrm{PS}_{1}$-entrer-ASSOC-PFV avec $\mathrm{AUG}_{1}-\mathrm{PN}_{1}$-jeune.fille et
umeengo ni janiíne
apparemment COP Jeanine
Il est entré avec une jeune fille, et apparemment c'est Jeanine!
(Nyerek'akaranga, Théâtre, 1970s)

Toutefois, la source inférentielle de l'évidentiel n'est pas nécessairement visuelle. Elle peut se baser sur tous les sens. Il s'agit généralement de l'ouïe, mais aussi de l'odorat, du toucher, etc., en résumé tout ce qui réfère au 
domaine du verbe -úmv- en kirundi. Ce verbe signifie non seulement « entendre », mais recouvre également les autres perceptions. En (5), la forme impérative de ce verbe invite l'interlocuteur à faire attention pour écouter. Comme en (2), l'évaluation épistémique se fonde sur l'ouïe : le locuteur croit avoir entendu celui qui arrive.

(5) Umve ca gikoko umengo araje Mungu wanje!

úmv-e ki-áa ki-kóokó umeengo a-ra-əz-ye

entendre-IMP $\mathrm{PP}_{7}-\mathrm{DEM}_{\mathrm{f}} \mathrm{PN}_{7}$-fauve apparemment $\mathrm{PS}_{1}$-DJ-venir-PFV

muúngu u-anje

Dieu $\quad \mathrm{PP}_{1}$-POSS 1 sg

Écoute, le fauve-là, apparemment il vient, mon Dieu !

(Nyerek'akaranga, Théâtre, 1970s)

Le verbe -úmv- renvoie aussi à la compréhension, touchant ainsi l'aspect intellectuel ou mental. Cette valeur évidentielle est d'autant plus indéniable que dans certains cas la phrase mentionne explicitement la présence d'un verbe cognitif sur laquelle l'évaluation du degré de certitude s'est fondée. C'est le cas en (6) où le locuteur se fonde sur le verbe -raab- qui se traduit généralement par " regarder », mais peut aussi faire référence à l'observation mentale, donc dans le sens de « évaluer».

(6) Turavye ivyo tuvuze, tuvyihweje dusanga umengo Iatrou Mikaeli nta gikorwa kiboneka yagize muri ico gikorwa.

\begin{tabular}{|c|c|c|}
\hline $\mathrm{tu}^{\mathrm{H}}$-raab-ye & i-bi-ó & tu-vúg- ${ }^{\mathrm{H}}$ ye \\
\hline $\begin{array}{l}\mathrm{PS}_{1 \mathrm{pl}} \cdot \mathrm{CJC} \text {-regarder-PFV } \\
\mathrm{u}^{\mathrm{H}} \text {-bi-îhweez-ye }\end{array}$ & $\begin{array}{r}\mathrm{AUG}_{8}-\mathrm{PP}_{8}-\mathrm{PRCS} \\
\text { tu-saang-a }\end{array}$ & $\begin{array}{c}\mathrm{PS}_{1 \mathrm{pl}} \text {-dire-REL.PFV } \\
\text { umeengo }\end{array}$ \\
\hline $\mathrm{S}_{1 \mathrm{pl}} \mathrm{CJC}-\mathrm{PO}_{8}$-examiner & $\mathrm{PS}_{1 \mathrm{pl}}$-trouve & IPFV \\
\hline
\end{tabular}

iatrou mikaeli ntaa ki-korwá ki-bónek- ${ }^{\mathrm{H}} \mathrm{a}$

Iatrou Michel COP.NEG PN PN$_{7}$-tâche PS $_{7}$-être.concret-REL.IPFV

a-á-gir- ${ }^{\mathrm{H}}$ ye murí i-ki-o ki-korwá

$\mathrm{PS}_{1}$-PE-faire-REL.PFV LOC $_{18} \quad \mathrm{AUG}_{7}-\mathrm{PP}_{7}-\mathrm{DEM}_{\mathrm{b}} \quad \mathrm{PN}_{7}$-œuvre

Si nous évaluons ce que nous venons de dire, si nous l'examinons, nous trouvons qu'apparemment Iatrou Michel, il n'y a aucune tâche concrète qu'il a fait dans cette œuvre.

(Ubuzima, Histoire, 1990s)

En (7), umengo est même renforcé par un évidentiel exprimé par le verbe principal, à savoir -se- « sembler », qui a les mêmes propriétés de passer de la comparaison à l'approximation. 
(7) Bamwe mbere bakibaza ngo « Mbega ico kirogorogo kivuga iki? » Abandi ngo : « Umengo riho asa n'uje kutumenyesha ibindi bimana bisha. "

ba-mwé mberé ba-ka-1-báz-a ngo

$\mathrm{PP}_{2}$-certain d'ailleurs $\mathrm{PS}_{2}$-SUBSEC-REFL-demander-IPFV QUOT

mbeéga i-ki-o ki-rógorógo ki-vúg-a iki

INTERR $\mathrm{AUG}_{7}-\mathrm{PP}_{7}$-DEM $\mathrm{DN}_{\mathrm{b}} \mathrm{PN}_{7}$-impudent $\mathrm{PS}_{7}$-dire-IPFV quoi

a-ba-ndi ngo umeengo rího a-se-a na

$\mathrm{AUG}_{2}-\mathrm{PP}_{2}$-autre QUOT apparemment plutôt $\mathrm{PS}_{1}$-sembler-IPFV avec

u-u-əz-ye ku-tu-meny-iish-a i-bi-ndi

$\mathrm{AUG}_{1}-\mathrm{PP}_{1}$-venir-PFV $\quad \mathrm{PN}_{15}-\mathrm{PO}_{1 \mathrm{pl}}$-connaître-CAUS-VF $\quad \mathrm{AUG}_{8}-\mathrm{PN}_{8}$-autre

bi-máana bi-shá

$\mathrm{PN}_{8}$-dieu $\mathrm{PP}_{8}$-nouveau

Certains d'ailleurs se sont demandés : « Cet impudent, qu'est-ce qu'il dit ? ». Les autres disaient : "Apparemment plutôt, il semble venir nous faire connaître d'autres nouveaux dieux. "

(UbwuzureBushasha, Religion, 1960s)

Les autres adverbes épistémiques seraient inappropriés dans tous les exemples allant de (3) à (7). En utilisant les autres adverbes épistémiques, le locuteur doute aussi. Mais ce qu'il y a de plus dans l'emploi de umeengo/umeenga, c'est que là le locuteur infère sur base d'un indice : soit qu'il infère à partir de ce qu'il vient de voir, de ce qu'il vient d'entendre, etc.

D'autre part, umeengo/umeenga s'emploie également lorsque l'incertitude se base sur un oubli, comme en (8), où le locuteur ne se rappelle plus le nombre de membres de sa famille tués et exprime cette approximation par umeenga.

(8) Harabaye amagume menshi cane. Ego na jewe narabuze abantu atari bake. Mu muryango iwanje umengo hapfuye abantu icumi na batanu. ha-á-ra-bá-ye a-ma-gúme ma-ínshi caane eegó na $\mathrm{PS}_{16}$-PE-DJ-être-PFV $\mathrm{AUG}_{6}-\mathrm{PN}_{6}$-crise $\mathrm{PP}_{6}$-beaucoup très oui aussi jéewé N-á-ra-búr-ye a-ba-ntu a-ta-ri ${ }^{\mathrm{H}}$ moi $\quad \mathrm{PS}_{1 \mathrm{sg}}$-PE-DJ-perdre-PFV $\mathrm{AUG}_{2}-\mathrm{PN}_{2}$-personne $\mathrm{PS}_{1}$-NEG-être.REL ba-ké mu mu-ryango i wáanjé umeengo $\mathrm{PP}_{2}$-peu $\mathrm{LOC}_{18} \quad \mathrm{PN}_{3}$-famille $\mathrm{LOC}_{19}$ chez.moi vraisemblablement ha-á-pfú-ye a-ba-ntu i-ø-cúmi na ba-taanu $\mathrm{PS}_{16}$-PE-mourir-PFV $\mathrm{AUG}_{2}-\mathrm{PN}_{2}$-personne $\mathrm{AUG}_{5}-\mathrm{PN}_{5}$-dix et $\mathrm{PP}_{2}$-cinq Il y a eu une très grande crise. Oui, moi aussi, j'ai perdu beaucoup de gens. Dans ma famille, vraisemblablement, il y aurait eu mort de quinze personnes. (Mushingantahe, Paix, 2000s) 
L'incertitude véhiculée par umeenga en (8) pourrait aussi être rendue par le conditionnel. On utiliserait alors la construction typique de la possibilité épistémique, c'est-à-dire l'auxiliaire -bá- « être », comportant l'affixe modal -oo-, suivi d'un verbe principal, au mode relatif (Bostoen et al. 2012); en (9). Cependant, l'emploi du conditionnel ne véhicule pas tout le sens induit de la présence de l'adverbe épistémique; il manque ce renvoi au souvenir incertain, qui, donc, fait figure de source de l'information sur laquelle se fonde le locuteur pour utiliser l'adverbe umeengo/umeenga. Cette information sur la source se perdrait aussi si on le remplace par un adverbe purement épistémique comme nkeeka ou ngirango. Le souvenir peut donc aussi être fondateur d'évidentialité, une source que la littérature ne décrit pas de façon précise. Nous traduisons ici umeenga par "vraisemblablement », faute d'équivalent plus approprié.

(9) Mu muryango iwanje hoba hapfuye abantu icumi na batanu.

$\mathrm{mu}$ mu-ryango i wáanjé ha-oo-bá-a

$\mathrm{LOC}_{18} \quad \mathrm{PN}_{3}$-famille $\mathrm{LOC}_{19}$ chez.moi $\mathrm{PS}_{16}$-MOD-être-VF

ha ${ }^{\mathrm{H}}$-á-pfú-ye a-ba-ntu i-ø-cúmi na ba-taanu $\mathrm{PS}_{16}$. $\mathrm{CJC}$-PE-mourir-PFV $\mathrm{AUG}_{2}-\mathrm{PN}_{2}$-personne $\mathrm{AUG}_{5}-\mathrm{PN}_{5}$-dix et $\mathrm{PP}_{2}$-cinq Dans ma famille, il y aurait eu mort de quinze personnes.

(phrase du corpus (8) transformée)

Le cas présenté en (10) fait intervenir une approximation due à un souvenir incertain, ce qui illustre encore une fois ce contenu dubitatif que met en lumière le mot umeengo/umeenga.

(10) Kandi ninaba nibuka neza, umengo n'icegera c'uwuserukira Amerika aha mu Burundi niho yarari.

kaándi ní-N-a-bá- ${ }^{\mathrm{H}} \mathrm{a} \quad \mathrm{N}^{\mathrm{H}}$-ibuk-a

par.ailleurs $\quad \mathrm{EV}^{2}-\mathrm{PS}_{1 \mathrm{sg}}$-HYP-être-REL.IPFV $\quad \mathrm{PS}_{1 \mathrm{sg}}$.CJC-se.rappeler-IPFV

néezá umeengo na i-ki-egeera ki-a

bien vraisemblablement même $\mathrm{AUG}_{7}-\mathrm{PN}_{7}$-adjoint $\mathrm{PP}_{7}$-CONN

2 Ce morphème préinitial ní- qui, en combinaison avec l'élément hypothétique -a- qui suit le préfixe verbal, entre dans la construction d'une proposition conditionnelle, a seulement été évoqué occasionnellement dans les descriptions du kirundi (Meeussen 1959, p. 115, Rodegem 1967, p. 50), mais n’a jamais été nommé. En nous inspirant de Dubois et al. (2007, p. 189), nous proposons de l'appeler « éventuel ». 
u-u-serukir-a

$\mathrm{AUG}_{1}-\mathrm{PP}_{1}$-représenter-IPFV

bu-ruúndi ni ha-ó ameeriká a-ha

$\mathrm{mu}$

Amérique $\mathrm{AUG}_{16}-\mathrm{PP}_{16} \cdot \mathrm{DEM}_{\mathrm{a}} \mathrm{LOC}_{18}$

a-á-ri

$\mathrm{a}^{\mathrm{H}}$-ri

\section{$\mathrm{PN}_{14}$-rundi COP $\mathrm{PP}_{16}$-SUBST $\mathrm{PS}_{1}$-PE-être $\mathrm{PS}_{1}$.CJC-être}

Par ailleurs, si je me rappelle bien, vraisemblablement même l'adjoint du représentant [= de l'ambassadeur] de l'Amérique ici au Burundi c'est là qu'il était.

(Ubuzima, Histoire, 1990s)

Umeengo/umeenga sert aussi à exprimer un doute ironique pour faire comprendre plutôt une assertion sans ambages. Il renforce même la valeur de vérité de l'énoncé. L'approximation sert un double but : outre celui de l'ironie, elle cherche aussi à éviter à son interlocuteur le désagrément d'une vérité trop crue ; l'approximation est employée comme procédé de courtoisie. Ainsi, l'exemple (11) rapporte un dialogue entre deux jeunes gens, Ino et Suza. Le jeune homme demande la main de la jeune fille. Dans un contexte pareil, il est hors de question qu'une jeune fille ne sache pas qu'elle a déjà accordé son cœur à quelqu'un d'autre. C'est donc une approximation qui sert à d'autres fins : elle est feinte. Pourtant ceci est très courant en kirundi et beaucoup d'exemples sont attestés dans le corpus.

(11) Ino - Nariko ndahiga umugenzi nkabaza ko uwogukunda bitokunda. Suza - Utabarije nk'ahandi umengo aha abandi baragutanze...
ino N-a-ri-kó
N-ra-híig-a
u-mu-geenzi

Ino $\mathrm{PS}_{1 \mathrm{sg}}$-PH-être-POSTF $17 \mathrm{PS}_{1 \mathrm{sg}}$-DJ-chercher-IPFV $\mathrm{AUG}_{1}-\mathrm{PN}_{1}$-amie

N-ka-báz-a

kó u-u-oo-ku-kúund-a

$\mathrm{PS}_{1 \text { sg }}$-SUBSEC-demander-VF que $\mathrm{AUG}_{1}-\mathrm{PP}_{1}-\mathrm{MOD}-\mathrm{PO}_{2 \mathrm{sg}}$-aimer-IPFV

bi-ta-oo-kúund-a

suza

$\mathrm{PS}_{8}$-NEG-MOD-être.possible-IPFV Suza

$\mathrm{u}^{\mathrm{H}}$-ta-báz-ir-ye nka a-ha-ndi

$\mathrm{PS}_{2 \mathrm{sg}}$.CJC-NEG-demander-APPL-PFV comme $\mathrm{AUG}_{16}-\mathrm{PP}_{16}$-autre

umeengo a-ha a-ba-ndi

vraisemblablement $\quad \mathrm{AUG}_{16}-\mathrm{PP}_{16} \cdot \mathrm{DEM}_{\mathrm{a}} \quad \mathrm{AUG}_{2}-\mathrm{PP}_{2}$-autre

ba-á-ra-ku-táang-ye

$\mathrm{PS}_{2}$-PE-DJ-PO ${ }_{2 \mathrm{sg}}$-devancer-PFV

Ino : J'étais en train de chercher une amie et je demande si celui qui t'aimerait, ce ne serait pas possible.

Suza : Si tu ne demandes pas (=tu ferais mieux de demander) ailleurs, vraisemblablement ici (= chez moi) d'autres t'ont devancé...

(NiAgasaga, Théâtre, 1960s) 
L'adverbe peut aussi être employé par courtoisie sans ironie. Le locuteur s'en sert pour faire comprendre que l'affirmation n'est pas un absolu, qu'il faut la considérer comme un humble avis du locuteur. Tel est le cas en (12).

(12) Jewe kuri jewe no kuri bagenzi banje, mbona umengo nta ngorane zoba zihari.

jeewé kurí jeewé nó kurí ba-geenzi ba-anje

moi $\mathrm{LOC}_{17}$ moi et $\mathrm{LOC}_{17} \mathrm{PN}_{2}$-ami $\mathrm{PP}_{2}$-POSS $\mathrm{POSg}_{1 \mathrm{sg}}$

N-bón-a umeengo ntaa N-goórane

$\mathrm{PS}_{1 \text { sg }}$-voir-VF vraisemblablement COP.NEG $\mathrm{PN}_{10}$-problème

zi-oo-bá- ${ }^{\mathrm{H}_{\mathrm{a}}} \quad$ zi $^{\mathrm{H}}$-ha-ri

$\mathrm{PS}_{10}$-MOD-être-REL.IPFV PS 10 .CJC-PO 16 -être

Moi, pour moi et pour mes amis, je vois que vraisemblablement il n'y aurait pas de problèmes.

(Mushingantahe, Paix, 2000s)

Il est indispensable de se demander à ce niveau si un tel emploi de umeengo/ umeenga peut être mis sur le compte d'une évolution post-modale. Nous n'irions pas jusque-là et dirions plutôt qu'il s'agit que d'un emploi pragmatique relevant du même sens épistémique, dans la mesure où il s'agit d'une stratégie $\mathrm{du}$ locuteur pour se positionner par rapport à son interlocuteur. En donnant à son propos une faible valeur de vérité, il affirme sa modestie à se rabaisser parmi les personnes dont les avis font peu autorité.

\section{De verbe à adverbe : grammaticalisation de umeengo/umeenga}

Tous les adverbes kirundi qui marquent la possibilité épistémique comportent un verbe conjugué comme composant historique (Mberamihigo 2014, pp. 275-335). C'est aussi le cas de umeengo/umeenga. Cette origine verbale a été reconnue par Ntahokaja (1994, p. 182) qui classe les formes umeengo/umeenga parmi ce qu'il appelle les " résidus de forme verbale ». Il réserve cette appellation à des « particules invariables [qui] se prêtent à un rapprochement avec des formes verbales par leur structure ou par leur construction ». Dans cette section, nous tentons de reconstruire le processus d'univerbation qui a mené à l'émergence de l'adverbe épistémique et évidentiel umeengo/umeenga, tout en considérant les légères variations de forme qu'il manifeste dans certaines variantes régionales du kirundi. 


\subsection{Univerbation et attrition phonologique de la locution umenya ngo}

Nous postulons ici que l'adverbe umeengo/umeenga résulte de la fusion entre la forme verbale umenya 'tu sais' et la conjonction ngo. Ce dernier est un complémenteur quotatif qui est également un des composants historiques de l'adverbe épistémique ngirango « peut-être ; probablement » (cf. infra). À la différence du verbe défectif -ti qui n'introduit que le discours direct (Cristini 2001 : 244) et du complémenteur kó qui n'introduit que le discours indirect, ngo peut introduire le discours direct ou indirect. Dans le cas de ngo « la parole ou la pensée est rapportée sans garantie de certitude', tandis que kó « présente la pensée ou la parole comme certaines » (Meeussen 1959 : 224). Le composant umenya est à l'origine une forme conjuguée de -meny- « connaître », un verbe de cognition très courant tant en kirundi qu'en bantou, comme en témoigne la reconstruction variable *-màny-/-mèny- "savoir " qui remonte ultimement au proto-bantou (Bastin et al. 2002). Le verbe est conjugué ici à la 2 sg $\left(\mathrm{PS}_{2 \mathrm{sg}}=\mathbf{u}-\right)$ au présent, ce dernier étant marqué par zéro en kirundi. La locution umenya ngo ne représente pas elle-même l'information la plus importante ou saillante que le locuteur veut passer à son interlocuteur ; celle-ci suit. Par conséquent, le verbe ne prend pas non plus la marque -ra- du 'disjoint'. Il s'agit d'un verbe dit 'conjoint' qui n'est pas marqué sur le plan morphologique (Bostoen \& Nshemezimana à paraître). Littéralement, umenya ngo signifierait donc « tu sais que », mais avec cette nuance que l'information qui suit n'est pas certaine (cf. Meeussen 1959 : 224). Toutefois, en kirundi dit commun, ce syntagme ne s'utilise plus, ni avec un sens lexical, ni avec un autre sens. Il n'est pas du tout attesté dans notre corpus, même pas dans les textes anciens. Par contre, comme illustré en (13), le syntagme umenya kó « tu sais que », où le complémenteur introduit une information considérée comme certaine (cf. Meeussen 1959 : 224), est bien présent dans notre corpus du kirundi dit commun.

(13) Ico gihe reeró shaáhu, uzooca uménya kó ubaayé umugabo.

$\begin{array}{lllll}\text { i-ki-o } & \text { ki-he } & \text { reeró shaáhu } & \text { u-zoo-ci-a } \\ \mathrm{AUG}_{7}-\mathrm{PP}_{7}-\mathrm{DEM}_{\mathrm{b}} & \mathrm{PN}_{7} \text {-moment donc } & \text { cher.ami } & \mathrm{PS}_{2 \mathrm{sg}} \text {-FUT-passer-IPFV } \\ \mathrm{u}^{\mathrm{H}} \text {-meny-a } & \text { kó } \mathrm{u} \text {-bá- }{ }^{\mathrm{H}} \text { ye } & \mathrm{u} \text {-mu-gabo } \\ \mathrm{PS}_{2 \text { sg. }} \text {.CJC-savoir-IPFV que } & \mathrm{PS}_{2 \text { sg }} \text {-être-REL.PFV } & \mathrm{AUG}_{1}-\mathrm{PN}_{1} \text {-homme }\end{array}$

En ce moment-là donc, cher ami, tu sauras alors que tu es devenu un homme.

(Es’iyo, Nouvelles, 1980s)

Bien que le syntagme umenya ngo ne soit plus présent en kirundi dit commun, il se rencontre encore dans certaines variétés régionales. C'est notamment le cas 
de celle de l'Imbo central, plus précisément dans la région de Buhonga, où Cristini (2001 : 245) rapporte une variante longue de l'adverbe épistémique umeengo, à savoir umenyango. En effet, comme des informateurs de la région ont pu nous le confirmer, le syntagme ne s'y trouve pas non plus avec son sens lexical, mais plutôt avec un usage proche de l'adverbe en kirundi dit commun, d'où notre choix pour l'écrire comme un seul mot. Comme montré en (14), umenyango marque ici à la fois la possibilité épistémique et l'évidentialité, comme c'est le cas pour umeengo/umeenga en kirundi dit commun.

(14) Jeewé naánje umenyango uwo mutí ndamaze kuwúkoreesha.

jeewé naánje umenyango u-u-o mu-tí

moi moi.aussi vraisemblablement $\mathrm{AUG}_{3}-\mathrm{PP}_{3}-\mathrm{DEM}_{\mathrm{b}} \mathrm{PN}_{3}$-médicament

N-ra-mar-ye ku-u-kóreesh-a

$\mathrm{PS}_{1 \text { sg }}$-DJ-finir-PFV $\mathrm{PN}_{15}-\mathrm{PO}_{3}$-employer-IPFV

Moi aussi, vraisemblablement ce médicament, je l'ai déjà employé.

(kirundi de Buhonga; élicitée)

Cette variété régionale de l'Imbo met donc en lumière que l'adverbe épistémique attesté en kirundi dit commun est le résultat d'une fusion de deux mots, à savoir umenya et ngo, la variante umeengo étant le résultat initial et jusqu'à présent la forme la plus utilisée (cf. supra). Dans le kirundi parlé dans la région de Buhonga, le premier stade de cette univerbation du verbe conjugué et du complémenteur peut encore être observé, à savoir la fusion avec maintien de la forme des deux composants historiques. En kirundi dit commun, par contre, ce processus s'est accompagné de la perte de la syllabe finale du verbe. Ce type d'attrition phonologique n'est pas courant en kirundi, mais s'observe souvent dans les langues $\mathrm{du}$ monde en cas d'univerbation et constitue un des changements formels communément associés à la grammaticalisation (Hopper \& Traugott 2003 : 154).

\subsection{Erosion sémantique de la forme issue de l'univerbation}

L'émergence de l'adverbe umeengo/umeenga peut non seulement être considérée comme un cas de grammaticalisation en raison de cette réduction phonologique par univerbation, mais aussi parce qu'il a subi une évolution de sens très caractéristique de la grammaticalisation, à savoir l'érosion sémantique ou « semantic bleaching ». Il s'agit ici d'un éloignement du sens lexical ou premier des différents composants vers un sens qui est non seulement non-compositionnel, mais aussi plus grammatical et plus général. Comme mentionné ci-dessus, ni dans le kirundi dit commun ni dans la variété de Buhonga, le verbe -meny- ne se 
rencontre actuellement en tant que verbe de cognition suivi de ngo, tandis que ce dernier ne fonctionne plus comme complémenteur quotatif. À part cela, le figement qui caractérise l'évolution de umeengo par univerbation se fait également par la désémantisation de u-. Dans l'adverbe univerbé, tant dans le kirundi dit commun que dans la variété de Buhonga, cet élément a perdu sa référence précise à la 2sg. Par conséquent, il n'est plus à considérer comme un préfixe sujet à part entière. La conséquence formelle logique de cette évolution sémantique est observée dans une autre variété du kirundi, à savoir le kibo, parlé dans la région de Cibitoke au nord-ouest. L'adverbe y existe sous forme raccourcie, c'est-à-dire dépourvue de sa voyelle initiale. On y dit plus fréquemment meengo/meenga.

\section{(15) Injiza ibikóreesho meengo haja kugwa imvúra nyiínshi.}

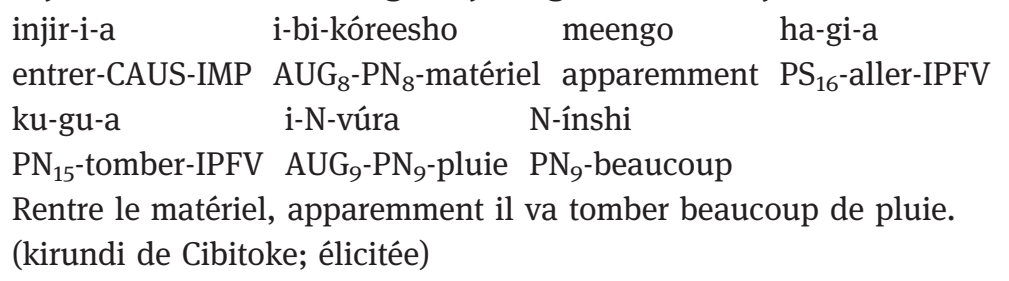

Le même mécanisme de désémantisation du $\mathrm{PS}_{2 \mathrm{sg}}$ s'observe dans le cas de l'adverbe épistémique ubóna " peut-être » dont une illustration est présentée en (16). Cet adverbe est issu du verbe -bón- " voir » et se traduirait littéralement par « si tu vois ». Son sens épistémique provient du fait qu'il peut être compris comme " on peut estimer ». La colline de recensement à laquelle le notable renvoie en (16) est, dans le vocabulaire administratif du Burundi, l'entité de base dans le découpage administratif du milieu rural qui regroupe au moins deux collines géographiques (Frey 1996 : 72).

(16) Umushingantahe : Ngirango ni uko ari ku mutumba wa rusansuma. Sipiriyano : Ngirango ubona.

u-mu-shíingantaáhe ngirango ni $\mathrm{u}$-ku-ó
$\mathrm{AUG}_{1}-\mathrm{PN}_{1}$-notable peut.être $\mathrm{COP} \mathrm{AUG}_{15}-\mathrm{PP}_{15}$-PRCS $\mathrm{PS}_{1}$-être.REL
$\mathrm{ku}$ mu-túmba $\mathrm{u}$-a $\quad$ ru-saansuma
$\mathrm{LOC}_{17} \mathrm{PN}_{3}$-colline $\mathrm{PP}_{3}$-CONN $\mathrm{NP}_{11}$-recensement
sipiriyáano ngirango ubóna
Cyprien peut.être probablement
Le notable : Peut-être, c'est parce que c'est sur la colline de recensement.
Cyprien : Peut-être, probablement.
(UmusoziKivyeyi, Paix, 1990s)


Le $\mathrm{PS}_{2 \mathrm{sg}}$ semble jouer un rôle privilégié dans l'émergence de l'épistémique en kirundi. Comme montré en (17), il peut également générer un sens épistémique en combinaison avec le verbe -saang- "trouver " sans être figé déjà comme adverbe. Dans cet usage, le verbe peut incorporer ou non le préfixe -oo- qui marque aussi la possibilité épistémique en kirundi (Bostoen et al. 2012). Que ce dernier soit présent ou non, le verbe -saang- conjugué à la 2sg exprime la possibilité épistémique. Dans le dialogue en (17), les deux emplois sont rapportés. Il s'agit d'un échange entre trois personnes au sujet du comportement anormal d'un jeune homme, et les interlocuteurs posent des hypothèses sur les raisons de ses accès de folie. La 2sg ne se rapporte à aucun des interlocuteurs, mais est plutôt liée à la forme habituelle de ce verbe dans son emploi épistémique, où 2 sg a la même fonction déictique que « on ». Par conséquent, ce qui se traduirait littéralement par « tu trouves que » ou " tu peux trouver que » signifie ici « il est/serait possible que »; «ce qu'on trouve » renvoie ici à « ce qui appartient au monde du possible ».

(17) - Urya usanga ari umuzimu. Ni umuzimu.

- Umuzimu wa he?

- Harya wosanga ari ibiyoga vyinshi yari yanyoye.

u-ryá u-sáang-a $\quad \mathrm{a}^{\mathrm{H}}$-ri $\quad$ u-mu-zimu

$\mathrm{PP}_{3}$ - $\mathrm{DEM}_{\mathrm{C}} \quad \mathrm{PS}_{2 \mathrm{sg}}$-trouver-VF $\mathrm{PS}_{1}$.CJC-être $\mathrm{AUG}_{3}-\mathrm{PN}_{3}$-esprit.des.ancêtres

ni u-mu-zimu u-mu-zimu

COP $\mathrm{AUG}_{3}-\mathrm{PN}_{3}$-esprit.des.ancêtres $\mathrm{AUG}_{3}-\mathrm{PN}_{3}$-esprit.des.ancêtres

u-a hé ha-ryá u-oo-sáang-a $\mathrm{a}^{-}{ }^{\mathrm{H}} \mathrm{ri}$

$\mathrm{PP}_{3}$-CONN où $\mathrm{PP}_{16}-\mathrm{DEM}_{\mathrm{c}} \mathrm{PS}_{2 \mathrm{sg}}$-MOD-trouver-VF $\mathrm{PS}_{1}$.CJC-être

i-bi-yogá bi-înshi a-a-ri ${ }^{\mathrm{H}} \quad \mathrm{a}^{\mathrm{H}}$-a-nyó-ye

$\mathrm{AUG}_{8}-\mathrm{PN}_{8}$-bière $\mathrm{PP}_{8}$-beaucoup $\mathrm{PS}_{1}$-PH-être.REL $\mathrm{PS}_{1}$.CJC-PE-boire-PFV

- Celui-là, c'est probablement un esprit des ancêtres. C'est un esprit des ancêtres.

- Un esprit d'où?

- Là, ce serait probablement (sous l'effet de) beaucoup trop de bière qu'il a bue.

(Igiti, Thêâtre, 2010s)

Le $\mathrm{PS}_{2 \mathrm{sg}}$ a en réalité la valeur d'un pronom neutre ou impersonnel dans ces contextes. Nous trouvons en kirundi d'autres emplois de ce genre. Ainsi, avec le verbe polysémique -gir- "faire, dire, penser ", nous remarquons qu'en s'adressant à un interlocuteur fictif, qui en réalité est tout destinataire de la phrase, il a 
un sens impersonnel. ${ }^{3}$ Cela correspond au contexte représenté en (18) où wogira est à comprendre dans le sens de « on pourrait penser ».

\section{(18) Wogira ngo abo bakenyezi b'imboneza ntabariho kuko batavugwa.}

u-oo-gir-a ngo a-ba-o ba-kényezi ba-a

$\mathrm{PS}_{2 \mathrm{sg}}$-MOD-penser-VF QUOT $\mathrm{AUG}_{2}-\mathrm{PP}_{2}-\mathrm{DEM}_{\mathrm{b}} \mathrm{PN}_{2}$-femme $\mathrm{PP}_{2}$-CONN

i-N-bonéza ntaa ba-ri ${ }^{\mathrm{H}}$-hó kukó

$\mathrm{AUG}_{10}-\mathrm{PN}_{10}$-leader COP.NEG $\mathrm{PP}_{2}$-être.REL-POSTF 16 parce.que ba-ta-vúg-u- ${ }^{\mathrm{H}} \mathrm{a}$

PS $_{2}$-NEG-parler-PASS-REL.VF

$\mathrm{Tu}$ pourrais (= on pourrait) penser que ces femmes leaders n'existent pas parce qu'on ne parle pas d'elles.

(CU101004Abaru, Paix, 2010s)

Il apparaît donc que la 2sg à laquelle on s'adresse au moment de la communication représente tous les interlocuteurs possibles dans leur entièreté, d'où la désémantisation de la personne dans ces verbes et adverbes épistémiques, où finalement le $\mathrm{PS}_{2 \mathrm{sg}}$ ne joue plus son rôle déictique. Cette érosion sémantique du $\mathrm{PS}_{2 \mathrm{sg}}$ fait donc partie du processus de transformation du verbe en adverbe. À cet égard, il faut cependant faire remarquer qu'un adverbe épistémique fait exception : ngirango « peut-être; probablement » illustré en (19). Cet adverbe est aussi né de l'univerbation d'un verbe qui peut renvoyer à la cognition, à savoir -gir- " faire, dire, penser », et le complémenteur ngo. Toutefois, le verbe n'est pas conjugué ici à la 2sg, mais à la 1sg. Toutefois, cette dernière a subi la même désémantisation.

(19) - Ni ya nyonko yawe idakira igarutse?

- Ni yo igarutse, ngirango.

ni i-áa N-nyoónko i-awe i-ta-kír- ${ }^{{ }^{H}}$

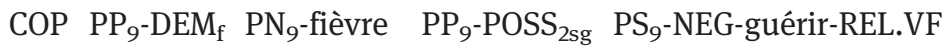

i-garuk- ${ }^{\mathrm{H}}$ ye ni i-ó i-garuk- ${ }^{\mathrm{H}}$ ye

$\mathrm{PS}_{9}$-revenir-REL.PFV COP $\mathrm{PP}_{9}$-SUBST $\mathrm{PS}_{9}$-revenir-REL.PFV

ngirango

probablement

- C'est ta fièvre incurable qui revient?

- C'est elle qui revient, probablement.

(Bugaboburihabwa, Nouvelles, 1990s)

3 L'infinitif kugira suivi de ngo s'est grammaticalisé comme un complémenteur introduisant une subordonnée de but en kirundi et kinyarwanda (Devos \& Bostoen 2012 : 123-4). 
En plus, ngirango n'a pas subi la réduction phonologique par l'apocope de la syllabe finale du verbe, mais par l'apocope du quotatif ngo même, comme illustré en (20).

(20) Ngira bayaze bumvikana kuko mbonye baherekeranije.

ngira ba-a-yaag-ye $\quad \mathrm{ba}^{\mathrm{H}}$-úmviikan-a

peut.être $\mathrm{PS}_{2}$-PH-échanger-PFV $\mathrm{PS}_{2}$.CJC-s'entendre-VF

kukó N-bón-ye $\quad$ ba $^{\mathrm{H}}$-hérekez-an-ye

parce.que $\mathrm{PS}_{1 \mathrm{sg}}$-voir-PFV $\mathrm{PS}_{2}$.CJC-accompagner-ASSOC-PFV

Peut-être, ils ont échangé dans l'entente parce que j'ai vu qu'ils s'accompagnaient l'un l'autre.

(Rekur'iyi, Théâtre, 1970s)

Le passage à la catégorie d'adverbe se caractérise aussi par une fluctuation tonale. Ce mot a des réalisations tonales qui varient essentiellement selon les locuteurs, et secondairement selon sa position dans la phrase. Par rapport aux locuteurs, l'influence géographique joue bien sûr un rôle, mais les divergences individuelles sont aussi un fait. Ce fait ne peut malheureusement pas être décrit dans tous ses détails à partir des données du corpus, dans la mesure où la grande majorité des textes constitutifs n'ont pas de tons pour des raisons liées aux habitudes orthographiques courantes du kirundi, le marquage des tons n'étant généralement limité qu'aux textes des milieux scolaire et académique. Néanmoins, à partir du peu de textes avec tons, nous pouvons exemplifier notre propos en évoquant les quatre façons d'écriture tonale retrouvées dans nos données : umeengó, umeengo, umeénga, umeenga. Toujours est-il qu'il existe un contexte où son schème tonal est prévisible : il s'agit des cas où elle est dans une position qui, si elle était occupée par un verbe, provoquerait pour celui-ci la présence du mode relatif, comme la conjonction kó « que ». Dans ces contexteslà on aurait umeengó/umeengá, avec toutefois la possibilité d'avoir les variantes individuelles umeéngo/umeénga.

Ces variations tonales indiquent que umeengo/umeenga manifeste une certaine indétermination entre les catégories adverbiale et verbale. Bien qu'on puisse lui attribuer le statut d'adverbe en tant que marque de la possibilité épistémique et de l'évidentialité, il garde néanmoins certaines propriétés d'un verbe. Ceci se révèle aussi dans les différentes positions syntagmatiques qu'il peut occuper dans la proposition. Sur le plan tonal, ce mot se comporte plutôt comme verbe lorsqu'il est placé avant la proposition dont il pronostique la valeur de vérité, mais comme adverbe lorsqu'il est après, ou lorsque, dans l'inutilité de répéter une information il apparaît seul dans une phrase. Comme le fait remarquer Ramat (2011 : 503) un adverbe ne peut représenter une phrase 
en elle-même que lorsqu'il est employé comme réponse à une question, comme c'est le cas dans le dialogue en (21). Ce comportement où umeengo acquiert une autonomie syntaxique est effectivement typique des adverbes et est aussi observé en (19), où ngirango se situe en position finale de la phrase, ce qui serait impossible si ngo fonctionnait toujours comme un complémenteur.

(21) Ikibazo : Yari yahunze?

Inyishu : Nti kwari uguhunga yari asanzwe ariyo ari.

Ikibazo : Akorerayo?

Inyishu : Umengo.

i-ki-bázo a-á-ri

$\mathrm{a}^{\mathrm{H}}$-a-huung-ye

$\mathrm{AUG}_{7}-\mathrm{PN}_{7}$-question

$\mathrm{PS}_{1}$-PE-être $\mathrm{PS}_{1}$.CJC-PH-fuir-PFV

i-N-ishú nti-ku-á-ri u-ku-huung-a

$\mathrm{AUG}_{9}$-PN $\mathrm{PN}_{9}$-réponse $\mathrm{NEG}-\mathrm{PS}_{15}$-PE-être $\mathrm{AUG}_{15}-\mathrm{PN}_{15}$-fuir-VF a-á-ri $\quad \mathrm{a}^{\mathrm{H}}$-saang-u-ye $\quad \mathrm{a}^{\mathrm{H}}$-ri i-ó

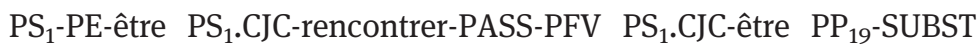

a-ri ${ }^{\mathrm{H}} \quad$ i-ki-bázo $\quad \mathrm{a}^{\mathrm{H}}$-kór-ir-a-yó

$\mathrm{PS}_{1}$-être.REL $\quad \mathrm{AUG}_{7}$-PN $\mathrm{PN}_{7}$-question PS $_{1}$.CJC-travailler-APPL-VF-POSTF 19

i-N-ishú umeéngo

$\mathrm{AUG}_{9}-\mathrm{PN}_{9}$-réponse semble-t-il

Question : Il avait fui?

Réponse : Ce n'était pas une fuite, c'est là qu’il travaillait normalement.

Question : Il y travaillait?

Réponse : Semble-t-il (/si mes souvenirs sont bons).

(Mushingantahe, Paix, 2000s)

Enfin, la variante umeenga de l'adverbe est à notre avis elle-même un indice de son statut flottant entre verbe et adverbe. C'est justement par analogie avec la voyelle finale par défaut des verbes en kirundi que la voyelle -o finale de umeengo a été réinterprété comme -a.

\section{D'adverbe à verbe : l'emploi comparatif de umeengo/umeenga}

L'usage de l'adverbe umeengo/umeenga ne se limite pas à l'évidentialité inférentielle illustrée dans la Section 3.2. Il s'utilise aussi comme un comparatif, comme nous le montrons ci-dessous à la Section 5.1. Des traces de ce même usage comparatif ont été trouvées dans des langues proches du kirundi, 
à savoir le kinyarwanda (JD61) et le kifuliiru (JD63). Remarquablement, comme nous le mettons en évidence à la Section 5.2, cet usage est attesté avec des formes non abrégées proches de celle à l'origine de umeengo/umeenga. Par contre, en kirundi même, utilisé ainsi, l'adverbe manifeste plutôt un comportement morphosyntaxique particulier dans certains contextes illustrés à la Section 5.3. Ceci suggère que l'adverbe est en train d'évoluer de nouveau vers un verbe, mais sous une forme qui est différente de sa forme verbale de départ.

\subsection{Usage de umeengo/umeenga en tant que comparatif}

Comme en témoigne l'exemple (22), l'adverbe peut s'employer aussi avec une valeur comparative fondée sur l'estimation dans la mesure où umeenga peut se traduire ici par " on dirait que ». Le locuteur observe l'attitude de son interlocuteur et la qualifie, en même temps qu'il la compare de façon métaphorique à une prise de distance " physique ».

(22) Nawe urabona ko bitoroshe, ariko umenga wigumira kure.

naáwe u-ra-bón-a kó bi-ta-óroh- ${ }^{\mathrm{H}}$ ye aríko

toi.aussi $\mathrm{PS}_{2 \mathrm{sg}}$-DJ-voir-IPFV que $\mathrm{PS}_{8}$-NEG-être.facile-REL.PFV mais

umeenga u-i-gum-ir-a kure

apparemment PS $_{2 \mathrm{sg}}$-REFL-rester-APPL-IPFV loin

Toi aussi tu vois que ce n'est pas facile, mais apparemment c'est comme si tu te tenais à distance.

(Kw'Isoko_2012_06, Religion, 2010s)

Dans cet emploi comparatif, umeengo/umeenga sert à exprimer un rapport de ressemblance entre deux éléments, faits ou situations qui se traduit par « cela ressemble à ", « cela est assimilé à... ", " c'est comme " ou " on dirait ". Il ne s'agit plus d'une expression de la probabilité. En (23), un tambourinaire chef d'orchestre debout au-devant de l'équipe déclame des paroles poétiques préliminaires au battement des tambours alignés. Il loue leur beauté. L’image des seins évoque les piquets fixés autour de la partie supérieure du tambour, servant à fixer et distendre la peau qui le recouvre, celle-ci constituant la surface de percussion.

(23) Ngizo zirasonze amabere umengo ni inkumi yo hambere basha!

ng-i-zi-ó zi-ra-soong-ye a-ma-béere

PRES-AUG $10-\mathrm{PP}_{10}$-DEM $\mathrm{DS}_{\mathrm{b}}$-DJ-être.pointu-PFV AUG $_{6}-\mathrm{PN}_{6}$-sein 
umeengo ni i-N-kumí i-ó haambere

c'est.comme $\mathrm{COP} \quad \mathrm{AUG}_{9}-\mathrm{PN}_{9}$-jeune.fille $\mathrm{PP}_{9}$-CONN jadis

baa shá

$\mathrm{PN}_{2 \mathrm{a}}$ ô.amis

Les voilà (les tambours) ils ont des seins pointus, on dirait une jeune fille de jadis, mes chers !

(Regards, Chansons, 1990s)

Tandis qu'en (23) la comparaison est établie sur une situation décrite par toute une proposition, en (24), le premier terme comparé est un simple syntagme nominal. Il ne s'agit pas d'une situation décrite, mais d'une chose : igitwé « une grosse tête ».

(24) Ico gitwe, umenga ni aho urupfu rwotera izuba!

i-ki-o ki-twé umeenga ni a-ha-ó

$\mathrm{AUG}_{7}-\mathrm{PP}_{7}-\mathrm{DEM}_{\mathrm{b}} \quad \mathrm{PN}_{7}$-tête c'est.comme COP AUG $\mathrm{AU}_{6}-\mathrm{PP}_{6}$-PRCS

u-ru-pfú ru-oot-ir- ${ }^{\mathrm{H}_{\mathrm{a}}}$ i- $\emptyset$-zúuba

$\mathrm{AUG}_{11}-\mathrm{PN}_{11}$-mort $\mathrm{PS}_{11}$-se.chauffer-APPL-REL.IPFV $\mathrm{AUG}_{5}-\mathrm{PN}_{5}$-soleil

Cette grosse tête, on dirait là où la mort vient se bronzer au soleil !

(Kutamenya, Théâtre, 2010s)

\subsection{Traces du comparatif en kinyarwanda et en kifuliiru}

Dans deux langues proches du kirundi, à savoir le kinyarwanda (JD61) et le kifuliiru (JD63), nous avons trouvé quelques traces de ce même usage comparatif, toutefois en association avec la forme longue qui a été postulée comme forme originelle de l'adverbe en kirundi. En kinyarwanda, le syntagme umenyango apparaît dans un poème guerrier rapporté par le traditionnaliste Alexis Kagame (1969), à qui nous devons aussi la traduction française reprise en (25). Une traduction plus littérale serait « Je tirai une flèche sur lui et il se renversa ; on ne put verser de l'eau sur lui (pour le ranimer); ce fut comme s’il avait été frappé par la foudre ». Un locuteur du kirundi aurait utilisé à la place les formes semi-conjuguées waméengo ou waméenga, dont nous traitons à la Section 5.3 ci-dessous.

(25) Ndamurasa arisenya ntiyasukirwa amazi, umenya ngo akubiswe n'inkuba.

N-ra-mu-rás-a a-ra-i-séeny-a

$\mathrm{PS}_{1 \text { sg }}$-DJ-PO $\mathrm{P}_{1}$-tirer.une.flèche-VF $\mathrm{PS}_{1}$-DJ-REFL-abattre-VF 
nti-a-a-suk-iir-a a-ma-azi

NEG-PS ${ }_{1}$-PH-verser-INTENS-VF AUG $_{6}$ - $\mathrm{PN}_{6}$-eau

umenyango a-kúbit-u-ye na i-N-kubá

comme.si $\quad \mathrm{PS}_{1}$-frapper-PASS-PFV par $\mathrm{AUG}_{9}-\mathrm{PN}_{9}$-foudre

Je lui décochai une flèche et il tomba de tout son long, sans qu'il fût possible de le ranimer, tel celui qu'aurait touché la foudre.

(kinyarwanda; Kagame 1969: 16-7)

Même si l'un des locuteurs du kinyarwanda interrogés à ce sujet connaissait par cœur le texte dont la phrase en (25) a été extraite, tous admettent ne plus utiliser cette forme. Ceci est peu surprenant, étant donné que Kagame (1969) précise que l'auteur du texte vivait à la cour royale autour de 1790 . Il s'agit donc d'un archaïsme.

La seule autre attestation de umenyango en kinyarwanda est rapportée en (26). Dans cet exemple, tiré d'une page web, il véhicule un sens épistémique et évidentiel tel qu'il a été décrit ci-dessus pour umeengo/umeenga en kirundi dit commun.

\section{(26) Umenya ngo irushanwa hari aho rigera bakambara bikini.}

umenyango i-rushanywa ha-ri

apparemment $\mathrm{AUG}_{5}$-compétition $\mathrm{PS}_{16}$-être

a-ha-ó ri-ger- ${ }^{\mathrm{H}}$ ba-ka-ambar-a bikini

$\mathrm{AUG}_{16}$-PP 16 -PRCS $\mathrm{PS}_{5}$-arriver-REL.VF $\mathrm{PS}_{2}$-SUBSEC-s'habiller-VF bikini

Dans la compétition il arrive apparemment un moment où elles s'habillent en bikinis.

(kinyarwanda; http://rushyashya.net/imyidagaduro/akineza-muri-15-baha tanira-kuba; consulté le 5/8/2014)

Il s'agit en (26) sans doute de l'emploi occasionnel d'une forme tombée en désuétude. Les locuteurs natifs du kinyarwanda interrogés préfèreraient utiliser ici ubaanza, traduit par " peut-être » dans le dictionnaire kinyarwanda de Coupez et al. (2005 : 2676) qui ne mentionnent umenya que comme son synonyme sans aucune autre information que sa catégorie d'appartenance. Les auteurs classent les deux mots comme " préverbe », avec comme définition un « mot qui précède un verbe et signifie qu'on doute sur ce que le verbe exprime », ce qui évoque la possibilité épistémique.

Quant au kifuliiru, Van Otterloo (2011) lui-même ne fait mention d'aucune forme qui soit apparentée à l'adverbe du kirundi. Néanmoins, nous avons été en mesure d'y retrouver, grâce à un locuteur natif, une forme similaire qui 
rappelle l'emploi comparatif de umeengo/umeenga en kirundi. Elle est rapportée en (27).

(27) Ilîira nyúumba wangamenya kwó mugina.

i-líira N-nyúumba u-anga-meny-a kwó mu-gina

$\mathrm{PP}_{9}$-DEM $\mathrm{PN}_{9}$-maison $\mathrm{PS}_{2 \text { sg }}$-POT-savoir-IPFV que $\mathrm{PN}_{3}$-termitière

Cette maison-là, on dirait une termitière.

(kifuliiro; élicitée)

Tout comme dans le dialecte kirundi parlé au Buhonga et en kinyarwanda, ce comparatif employé en kifuliiru se base sur une forme conjuguée du verbe -meny« savoir ». En outre, il prend comme sujet celui de la 2sg à l'instar de ce que nous avons observé pour le kirundi. Néanmoins, il diffère du kirundi sous plusieurs aspects. Premièrement, étant donné que umeengo/umeenga n'existe pas tel quel en kifuliiru, pour avoir le même sens comparatif qu'en kirundi, le kifuliiru doit ajouter impérativement l'affixe potentiel, qui est facultatif en kirundi. D'autre part, le kirundi ajoute le verbe " être » ou une copule avant le substantif qui sert de complément à umeengo/umeenga (cf. infra), ce qui n'est pas le cas en kifuliiru. Enfin, il n'y est pas employé comme épistémique évidentiel. Il est employé uniquement comme un comparatif, ce qui correspond à ce qu'on observe en kinyarwanda archaïque. Le fait que dans des langues proches du kirundi ce sens est associé à la forme non encore abrégée, voire au verbe non encore figé en kifuliiru, suggère qu'il n'est pas nécessairement le résultat d'un changement sémantique ultérieur en kirundi même, mais qu'il peut avoir été lié à cette forme avant même son univerbation et l'attrition phonologique qui s'en est suivie. L'occurrence de cette fonction en association avec la forme longue dans certaines variétés régionales du kirundi comme en (28), en donne la preuve.

(28) Raaba ingéne kárya gasózi gashiinzé; kukáduuga umenyango ni ukwuúrira igití.

raab-a ingéne ka-ryá ka-sózi ka-shiing- ${ }^{\mathrm{H}}$ ye regarder-IMP comment $\mathrm{PP}_{12}-\mathrm{DEM}_{\mathrm{c}} \mathrm{PN}_{12}$-colline $\mathrm{PS}_{12}$-être.raide-REL.PFV

ku-ka-dúug-a umenyango ni u-ku-úrir-a

$\mathrm{PN}_{15}-\mathrm{OBJ}_{12}$-monter-IPFV c'est.comme.ci COP $\mathrm{AUG}_{15}-\mathrm{PN}_{15}$-grimper-IPFV

i-ki-tí

$\mathrm{AUG}_{7}-\mathrm{PN}_{7}$-arbre

Regarde comment cette colline est raide, la monter, c'est comme grimper sur un arbre.

(kirundi de Buhonga; élicitée) 


\subsection{Evolution morphosyntaxique du comparatif umeengo/umeenga}

Par rapport à l'adverbe épistémique et évidentiel, la forme qui exprime le comparatif semble avoir subi une évolution morphosyntaxique ultérieure vers une nouvelle forme verbale qui se distingue du verbe d'origine -meny-. Dans son emploi comparatif, umeengo/umeenga a un statut flottant entre celui d'un adverbe et celui d'un verbe défectif. Il peut adopter des marqueurs de temps et de mode à l'instar d'un verbe. Pour ce qui est de l'expression du temps, il s'agit avant tout du passé hodiernal (-a-) et du passé éloigné (-á-), étant entendu que la marque du présent est zéro en kirundi. Le corpus n'atteste aucun cas du futur -zoo-. À notre connaissance il est inusité en combinaison avec umeengo/umeenga. En plus, aucune langue voisine analysée ni aucune variante régionale n'en font état. L'insertion de la marque du passé éloigné dans la forme à finale -o est illustrée en (29), et en (30) pour celle à finale -a. ${ }^{4}$ Le fait que la voyelle -o finale du mot umeengo reste en (29), même lorsque ce mot se comporte comme un verbe, subissant des opérations liées à la conjugaison, indique que ce nouvel usage verbal a bien émergé d'une forme complexe d'origine mixte, la voyelle provenant donc de la finale du quotatif ngo.

(29) Wa musore na ya nkumi wamengo ni umwami n'umwamikazi bagiye mu birori.

u-áa mu-sóre na i-áa N-kumí

$\mathrm{PP}_{1}$-DEM $\mathrm{f} \quad \mathrm{PN}_{1}$-jeune.homme et $\mathrm{PP}_{9}$ - $\mathrm{DEM}_{\mathrm{f}} \mathrm{PN}_{9}$-jeune.fille

u-á-meengo ni u-mu-aámi na u-mu-aámikazi

$\mathrm{PS}_{2 \mathrm{sg}}$-PE-croire COP $\mathrm{AUG}_{1}-\mathrm{PN}_{1}$-roi et $\mathrm{AUG}_{1}-\mathrm{PN}_{1}$-reine

ba-gi- ${ }^{\mathrm{H}} \mathrm{ye} \quad \mathrm{mu}$ bi-rori

$\mathrm{PS}_{2}$-aller-REL.PFV $\mathrm{LOC}_{18} \quad \mathrm{PN}_{8}$-cérémonie

Ce jeune homme-là et cette jeune fille-là, on aurait dit un roi et une reine qui vont à des cérémonies.

(NiAgasaga, Théâtre, 1960s)

(30) Narakubiswe ga sha; wamenga n'imbwa yivye ifashwe ikubitwa.

N-á-ra-kúbit-u-ye

ga sha

u-á-meenga

ni

$\mathrm{PS}_{1 \text { sg }}$-PE-DJ-battre-PASS-PFV VOC mon.ami $\mathrm{PS}_{2 \mathrm{sg}}$-PE-être.comme COP i-N-bwá i-îb- ${ }^{\mathrm{H}}$ ye i-fát-u- ${ }^{\mathrm{H}}$ ye

$\mathrm{AUG}_{9}$-PN $\mathrm{PN}_{9}$-chien $\mathrm{PS}_{9}$-voler-REL.PFV $\mathrm{PS}_{9}$-attraper-PASS-REL.VF

4 L'intégration de la marque du passé hodiernal s'observe dans l'exemple (42) qui suit. 


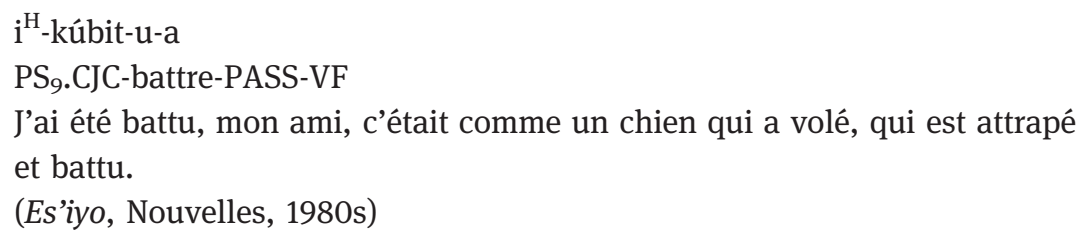

Cette évolution de umeengo/umeenga vers un nouveau statut verbal est vraiment liée à son usage comparatif mais est exclue quand l'adverbe marque la possibilité épistémique et l'évidentialité. Comme montré en (10) ci-dessus, dans un emploi qui correspond à une situation du passé, la référence temporelle est alors portée par le verbe de la phrase, tandis que umeengo demeure invariable. S’il avait subi un ajustement temporel, l'on serait retombé dans le sens comparatif, ce qui se passe en (31). La mise au passé de cette forme lui donne automatiquement un sens comparatif.

(31) Ipataro n'ishati yari yagoroye wamengo yaremanywe n'umuraba.

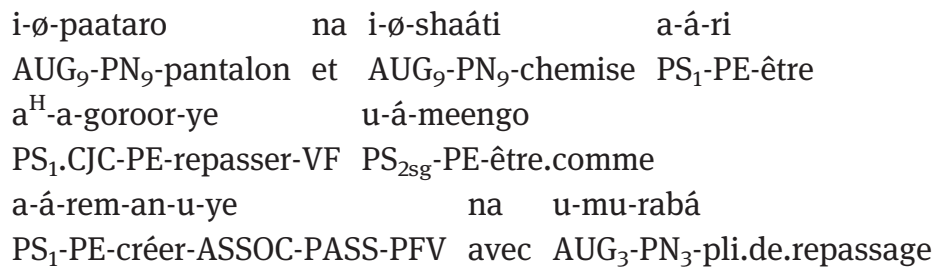

Le pantalon et la chemise, il avait repassé, on aurait dit qu'il avait été créé avec le pli de repassage.

(Umwigeme, Nouvelles, 1970s)

En ce qui concerne les modes matérialisés par des affixes spécifiques, umeengo/umeenga connaît la forme du potentiel marquée par -oo-, et la forme subsécutive marquée par -ka-. Dans un cas comme dans l'autre, le choix de la voyelle finale -o ou -a demeure arbitraire. En (32) et (33), les deux variantes portent en tant que verbe défectif le préfixe -oo- du mode potentiel. Dans ce cas, il décrit une apparence, souvent à l'aide d'une image dans le sens de « on dirait que ». De par la présence de cet affixe modal, il indique que cette apparence est approximative, que le rapprochement est de l'ordre de la potentialité, qu'il y a à peu près similitude.

(32) Kăndi iyó gicănye gikūnda gutúragurika wōmēngo ni umūntu aríko arōtsa ibigôri.
kaándi iyó
ki-cáan- ${ }^{\mathrm{H}}$ ye
ki-kúund-a
en.outre lorsque
$\mathrm{PS}_{7}$-mettre.au.feu-REL.PFV
$\mathrm{PS}_{7}$-aimer-IPFV 
ku-túragurik-a u-oo-meengo ni u-mu-ntu

$\mathrm{PN}_{15}$-crépiter-VF $\mathrm{PS}_{2 \mathrm{sg}}$-MOD-ressembler $\mathrm{COP} \quad \mathrm{AUG}_{1}-\mathrm{PN}_{1}$-personne

a-ri ${ }^{\mathrm{H}}$-kó a-ra-ots-a i-bi-góori

$\mathrm{PS}_{1}$-être.REL-POSTF $17 \quad \mathrm{PS}_{1}$-DJ-griller-IPFV $\quad \mathrm{AUG}_{8}-\mathrm{PN}_{8}$-maïs

En outre, lorsqu'il est mis au feu il crépite généralement, on dirait une personne qui est en train de griller du maïs.

(IbitiNibiterwa, Culture Traditionnelle, 2010s)

(33) Umuyókayóka : Ni ibitěrwa birāndárānda hāsí, kăndi ahó biryāmye wōmēnga ni imitwé y’înzúzi.

u-mu-yókayóka ni i-bi-teérwa

$\mathrm{AUG}_{3}-\mathrm{PN}_{3}$-Cassia.occidentalis $\mathrm{COP} \quad \mathrm{AUG}_{8}-\mathrm{PN}_{8}$-plante

bi-ráandaraand- ${ }^{\mathrm{H}}$ haasí kaándi a-ha-ó

$\mathrm{PS}_{8}$-ramper-REL.IPFV par.terre et $\quad \mathrm{AUG}_{16}-\mathrm{PP}_{16}$-PRCS

bi-ryáam- ${ }^{\mathrm{H}}$ ye u-oo-meenga ni

$\mathrm{PS}_{8}$-être.couché-REL.PFV PS 2sg -MOD-ressembler COP

i-mi-twé i-ó i-N-yúzi

$\mathrm{AUG}_{4}-\mathrm{PN}_{4}$-bourgeon $\mathrm{PP}_{4}$-CONN $\mathrm{AUG}_{10}-\mathrm{PN}_{10}$-courge

Le Cassia occidentalis ${ }^{5}$ : ce sont des plantes qui rampent au sol, et là où elles sont couchées, on dirait que ce sont des bourgeons de courges.

(IbitiNibiterwa, Culture Traditionnelle, 2010s)

Quant à la forme subsécutive, elle est employée lorsqu'après avoir évoqué une situation, l'on fait recours à une comparaison pour l'expliciter, celle-ci étant conçue comme une image explicative subséquente. Les deux formes sont illustrées en (34) et (35).

(34) Hari abakuru b'imirwi n'imihari bayigize icibare, ukamengo ni agatongo barazwe na ba sekuru.

\begin{tabular}{|c|c|}
\hline a-ba-kurú & i-mi-rwi \\
\hline $\mathrm{PS}_{16}$-être $\mathrm{AUG}_{2}-\mathrm{PN}_{2}$-chef & ef $\mathrm{PP}_{2}-\mathrm{CONN} \mathrm{AUG}_{4}-\mathrm{PN}_{4}$-groupe et \\
\hline i-mi-hari & ba-á-i-gir-ye $\quad$ i-ki-íbare \\
\hline $\begin{array}{l}\mathrm{AUG}_{4}-\mathrm{PN}_{4} \text {-mouvement } \\
\text { u-ka-meengo }\end{array}$ & $\begin{array}{l}\mathrm{PS}_{2} \text {-PE-REFL-faire-PFV } \mathrm{AUG}_{7}-\mathrm{PN}_{7} \text {-champ.réservé } \\
\text { ni a-ka-toongo }\end{array}$ \\
\hline $\mathrm{PS}_{2}$-SUBSEC-ressembler & COP $\mathrm{AUG}_{12}-\mathrm{PN}_{12}$-propriété \\
\hline $\begin{array}{l}\text { a-a-rag-u-ye } \\
S_{2} \text {-PE-léguer-PASS-PFV }\end{array}$ & 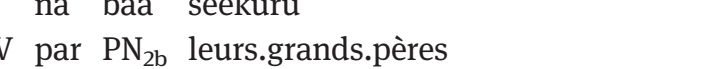 \\
\hline
\end{tabular}

5 L’identification scientifique de cette plante est donnée par Rodegem (1970 : 637). 
Il y a des chefs de groupes et de mouvements qui en ont fait un champ réservé, et ainsi c'est comme si c'est une petite propriété qu'ils ont reçu en héritage de la part de leurs grands-pères.

(Kw'Isoko_2011_33, Religion, 2010s)

(35) Raba nk'iyo serugo canke inarugo afata umukozi nabi ukamenga si umuntu nk'abandi.

raab-a nka iyó séerugó canké inárugó

regarder-IMP comme lorsque maître.de.céans ou maîtresse.de.céans

a-fát- ${ }^{\mathrm{H}_{\mathrm{a}}}$ u-mu-kózi náabí u-ka-meenga

$\mathrm{PS}_{1}$-prendre-REL.VF $\quad \mathrm{AUG}_{1}$ - $\mathrm{PN}_{1}$-travailleur mal $\mathrm{PS}_{2 \mathrm{sg}}$-SUBSEC-ressembler si u-mu-ntu nka a-ba-ndi

COP.NEG AUG $\mathrm{ANN}_{1}$-personne comme $\mathrm{AUG}_{2}-\mathrm{PP}_{2}$-autre

Regarde, par exemple lorsque le maître de céans ou la maîtresse de céans maltraite le travailleur, comme si ce n'était pas une personne comme d'autres.

(CU110308Umuro, Paix, 2010s)

Parmi les modes matérialisés par le ton, le conjonctif et le relatif peuvent être affectés à cette forme. Le conjonctif est marqué par un ton haut sur le sujet verbal. Son rôle est de référer à une action associée à une autre, d'où l'idée de conjonction, exprimant ainsi la conditionnalité, la simultanéité, l'opposition, la concession, etc. Il est aussi associé aux verbes dits conjonctifs, comme kubóna " voir », gusáanga " rencontrer, trouver », gusíga " laisser », kwuúmva " entendre, sentir »; il survient également après l'adverbe nkeeka " peut-être ", ou après certains auxiliaires comme kubá " être » (Meeussen 1959 : 109, Cristini 2001 : 166-7). La phrase en (36) illustre l'usage de umeenga en mode conjonctif.

(36) Uwo musi narabuze ico mfata n'ico ndeka; eka numva umenga si nkiri umugabo mu rugo.

\begin{tabular}{|c|c|}
\hline mu-si & N-á-ra-búr-ye \\
\hline $\mathrm{AUG}_{3}-\mathrm{PP}_{3}-\mathrm{DEM}_{\mathrm{b}} \mathrm{PN}_{3}$-jour & ir $\mathrm{PS}_{1 \mathrm{sg}}$-PE-DJ-manquer-PFV $\mathrm{AUG}_{7}-\mathrm{PP}_{7}$-PRCS \\
\hline $\mathrm{N}$-fát- ${ }^{\mathrm{a}} \mathrm{a}$ & a i-ki-ó $\quad$ N-rek- ${ }^{\mathrm{H}_{\mathrm{a}}}$ \\
\hline $\mathrm{PS}_{1 \mathrm{sg}}$-prendre-REL.VF & $\mathrm{AUG}_{7}-\mathrm{PP}_{7}-\mathrm{PRCS} \quad \mathrm{PS}_{1 \mathrm{sg}}$-laisser-REL.VF \\
\hline eka N-á-umv-a & $\mathrm{u}^{\mathrm{H}}$-meenga \\
\hline en.vérité $\mathrm{PS}_{1 \mathrm{sg}}$-PE-sentir- & -VF PS $_{2 \text { sg. }}$ CJC-être.semblable.à \\
\hline si-N-ki-ri & -mu-gabo \\
\hline NEG-PS ${ }_{1 \mathrm{sg}}$-PERST-être AU & $\mathrm{UG}_{1}$-PN $\mathrm{PN}_{1}$-homme $\mathrm{LOC}_{18} \quad \mathrm{PN}_{11}$-ménage \\
\hline
\end{tabular}


Ce jour-là j'ai manqué que prendre et que laisser, en vérité je sentais que c'était comme si je n'étais plus un homme dans le ménage.

(CU111124Inyota, Paix, 2010s)

Quant au mode relatif, il est caractérisé par un ton haut post-radical. Celui-ci se réalise généralement sur la voyelle finale, mais il est gouverné par des règles morpho-tonologiques complexes dans un verbe contenant des extensions verbales, qui s'intercalent précisément entre le radical et la finale. Quant à sa valeur, il est d'abord employé dans la proposition relative et dans une construction à précessif, mais il se retrouve dans de nombreux autres contextes : dans le verbe suivant le complémenteur kó « que », ou sa variante yúukó, de même qu'après kukó « parce que », et dans la proposition conditionnelle, où il s'associe avec le marqueur du mode potentiel. Le précessif est un type de pronom dont la fonction est de servir d'antécédent à une proposition relative objective, c'est-àdire qui n'est pas en relation d'accord avec son antécédent, le verbe pouvant être précédé de son propre sujet, à l'instar des relatives françaises référées par « que », « où », « dont » (Meeussen 1959 : 97). Dans le cas du mode relatif, que la forme en question soit terminée par -a ou par -o, ces dernières supportent le ton grammatical post-radical qui normalement frappe la voyelle finale pour le mode relatif. C'est notamment le cas lorsqu'il suit le complémenteur kó, ou le précessif ukó, ou un pronom relatif. L'exemple (37) illustre comment il se comporte en tant que verbe au mode relatif. Il a été extrait d'un texte du corpus comportant des marques tonales. Ainsi le phénomène peut être observé de façon directe.

(37) Amababi yâco aratândukānye kāndi ku bishíshwa harikó utūntu umēngó ni ubwǒya kăndi tuja turavūngagurika.

a-ma-babi a-áaco a-ra-táandukaan-ye kaándi

$\mathrm{AUG}_{6}-\mathrm{PN}_{6}$-feuille $\mathrm{PP}_{6}$-POSS $\mathrm{PS}_{6}$-DJ-être.différent-PFV et

$\mathrm{ku}$ bi-shíshwa ha-ri-hó u-tu-ntu

LOC $_{17} \quad \mathrm{PN}_{8}$-écorce $\mathrm{PS}_{16}$-être-POSTF $16 \quad \mathrm{AUG}_{13}-\mathrm{PN}_{13}$-chose

u-meengo $^{\mathrm{H}}$ ni u-bu-oóya kaándi tu-gi- ${ }^{\mathrm{H}} \mathrm{a}$

$\mathrm{PS}_{2 \text { sg }}$-ressembler.REL COP $\mathrm{AUG}_{14}-\mathrm{PN}_{14}$-poil et $\mathrm{PS}_{13}$-aller-REL.VF

tu-ra-vuung-agur-ik-a

$\mathrm{PS}_{13}$-DJ-s'émietter-INTENS-IMPO-VF

Ses feuilles sont écartées et sur l'écorce il y a des choses dont on dirait que ce sont des poils et elles s'émiettent continuellement.

(IbitiNibiterwa, Culture Traditionnelle, 2010s)

Le comparatif est attesté aussi sous une forme négative. Celle-ci survient dans un contexte stylistique particulier, où une forte affirmation procède par une 
formule de juron suivie d'une phrase négative. Celle-ci est généralement exclamative parce qu'elle fait part d'un certain émerveillement. En plus, umeengo/ umeenga comporte un ton haut post-radical caractéristique du mode relatif, comme illustré en (38).

\section{(38) Emwe nukuri data ubuho utamengo n'ivumwe!}

emwé ni u-ku-rí daáta ubu ha-ó

ah COP $\mathrm{AUG}_{15}-\mathrm{PN}_{15}$-vérité mon.cher actuellement $\mathrm{PP}_{16}$-SUBST

u-ta-meengo $^{\mathrm{H}}$ ni i-vumwe

$\mathrm{PS}_{2 \text { sg }}$-NEG-ressembler.à.REL COP $\mathrm{AUG}_{5}$-malédiction

Ah ! C'est la vérité [= Je vous jure, je vous assure] mon cher [= papa], actuellement en particulier, c'est comme [ = on dirait] une malédiction !

(Ndongozi5506Karusi, Information, 1950s)

Dans l'illustration présentée en (38), le locuteur s'exclame pour ce qui vient de lui arriver et pour renforcer ses propos, il lance un des jurons les plus courants en kirundi. La forme umeengo comporte alors une négation. Notons cependant que cette structure négative et ce mode relatif sont facultatifs dans ces phrases déclaratives avec présence de juron. En (39), nous présentons un exemple où la même formule de juron est employée sans pour autant qu'il y ait présence d'une forme négative de umeengo/umeenga. La négation a en effet pour rôle de renforcer la vérité de l'assertion ; quand ce besoin d'insistance n'est pas ressenti par le locuteur, la phrase demeure à l'affirmatif. Cela renforce notre propos selon lequel cette négation de umeengo/umeenga est d'ordre stylistique.

(39) Ku mutumba wa Murambi, ni ukuri amagume yarahashitse.

ku mu-tuúmba u-a muraámbi ni u-ku-rí

$\mathrm{LOC}_{17} \mathrm{PN}_{3}$-colline $\mathrm{PP}_{3}$-CONN Murambi COP $\mathrm{AUG}_{15}-\mathrm{PN}_{15}$-vérité

a-ma-gúme a-á-ra-ha-shik-ye

$\mathrm{AUG}_{6}-\mathrm{PN}_{6}$-trouble $\mathrm{PS}_{6}$-PE-DJ-PO 16 -arriver-PFV

Sur la colline de Murambi, en vérité, les troubles y sont arrivés.

(Mushingantahe, Paix, 2000s)

Comme plusieurs exemples qui précèdent en témoignent, le comparatif umeengo/umeenga a un statut semi-verbal, puisqu'il peut incorporer différents affixes flexionnels verbaux qui sont commutables. Un autre indice de son évolution en cours vers un statut plus verbal est le fait qu'au sein d'une construction à auxiliaire il peut occuper la position de l'auxilié derrière l'auxiliant. C'est le cas par exemple dans une construction à auxiliaire introduite par le verbe -cí- « passer » qui véhicule en tant qu'auxiliant le sens grammatical de 
postériorité, de succession, comme illustré en (40), où il se combine avec le verbe lexical -jaana « aller/partir ensemble avec ».

(40) Yaciye ampa umuduga, duca turajana n'umuzungu.

a-á-ci-ye $\quad \mathrm{a}^{\mathrm{H}}$-N-há-a u-mu-dúga

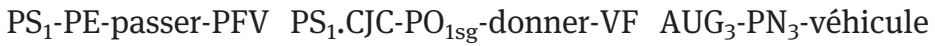
tu-ci-a tu-ra-gi-an-a na u-mu-zuúngu

$\mathrm{PS}_{1 \mathrm{pl}}$-passer-VF $\mathrm{PS}_{1 \mathrm{pl}}$-DJ-aller-ASSOC-VF avec $\mathrm{AUG}_{1}-\mathrm{PN}_{1}$-Européen

Ensuite il me donna un véhicule, et alors nous partîmes avec l'Européen.

(Mushingantahe, Paix, 2000s)

Il arrive que ce même auxiliant soit combiné à umeengo de façon que ce dernier se retrouve dans la position syntaxique occupée normalement par le verbe principal ou auxilié avec le même effet sémantique que celui décrit précédemment. Tout comme l'auxiliant -cí- prend la même marque de personne que l'auxilié en (40), à savoir le $\mathrm{PS}_{1 \mathrm{pl}}$, il adopte le $\mathrm{PS}_{2 \mathrm{sg}}$ d'umeengo en (41). Toutefois, contrairement à ce qu'on observe en (40), le $\mathrm{PS}_{2 \mathrm{sg}}$ ne renvoie pas ici à un véritable participant au discours. Il s'interprète sémantiquement toujours comme impersonnel.

(41) Aho lero Ndayiragije yabohwa bari bamaze kwibaruka umwana umwe w'umuhungu, bamwita Nzirubusa. Avutse, uca umengo ni we yatongereye se ngo bamujane mu mbohero.

a-ha-o reeró ndayiragije a-á-bóh-u- ${ }^{\mathrm{H}_{\mathrm{a}}}$

$\mathrm{AUG}_{16}-\mathrm{PP}_{16}-\mathrm{DEM}_{\mathrm{b}}$ alors Ndayiragije $\mathrm{PS}_{1}$-PE-emprisonner-PASS-REL.VF

ba-á-ri $\quad$ ba $^{\mathrm{H}}$-mar-ye ku-íbaruk-a u-mu-áana

$\mathrm{PS}_{2}$-PE-être $\mathrm{PS}_{2}$. CJC-finir-PFV $\mathrm{PN}_{15}$-mettre.au.monde-VF $\mathrm{AUG}_{1}$ - $\mathrm{PN}_{1}$-enfant

u-mwé u-a u-mu-huúngu ba-mu-it-a nzirúbusá

$\mathrm{PP}_{1}$-un $\mathrm{PP}_{1}$-CONN $\mathrm{AUG}_{1}-\mathrm{PN}_{1}$-garçon $\mathrm{PS}_{2}-\mathrm{PO}_{1}$-appeler-VF nzirúbusá

$\mathrm{a}^{\mathrm{H}}$-vúuk-ye u-ci-a u-meengo

$\mathrm{PS}_{1}$.CJC-naître-PFV $\mathrm{PS}_{2 \mathrm{sg}}$-passer-VF $\mathrm{PS}_{2 \mathrm{sg}}$-ressembler

ni u-é a-á-tongeer- ${ }^{\mathrm{H}}$ ye sé

COP $\mathrm{PP}_{1}$-SUBST $\mathrm{PS}_{1}$-PE-faire.des.imprécations-REL.PFV son.père

ngo ba-mu-gi-an-e mu N-bóhero

pour.que $\mathrm{PS}_{2}-\mathrm{PO}_{1}$-aller-ASSOC-SBJV $\mathrm{LOC}_{18} \quad \mathrm{PN}_{9}$-prison

Alors lorsque Ndayiragije fut emprisonné, ils avaient déjà mis au monde un enfant de sexe masculin, ils l'avaient appelé Nzirubusa. Quand il naquit, ce fut comme si c'était lui qui avait fait des imprécations sur son père pour qu'on le mette en prison.

(UmuganiWaNdayiragije, Contes, 1970s) 
Sur le plan morphologique, le comparatif umeengo/umeenga a donc plutôt l'allure d'un verbe polymorphémique commençant par un préfixe sujet que d'un adverbe monomorphémique. Sur le plan sémantique, cependant, l'élément $\mathbf{u}$ - ne s'est pas resémantisé comme une marque du 2sg. Dans aucun des exemples précédents, il ne renvoie de nouveau à un interlocuteur dûment identifié.

Par contre, une étape plus avancée de cette mutation vers un emploi verbal est marquée par la possibilité de faire usage d'un segment initial autre que u-, plus précisément la nasale homorganique $\mathbf{N}$-qui fonctionne bien comme un $\mathrm{PS}_{1 \mathrm{sg}}$, comme illustré en (42).

(42) Babarira umugabekazi, bati Bihore, ntibamenye k'unywa inzoga y’impeke. Gira urtya, uti namenga s’impeke.

ba-bárir-a u-mu-gabékazi ba-ti bi-hór-e

$\mathrm{PS}_{2}$-dire-VF $\mathrm{AUG}_{1}$ - $\mathrm{PN}_{1}$-reine.mère $\mathrm{PS}_{2}$-QUOT $\mathrm{PO}_{8}$-taire-IMP

nti-ba-meny-e kó u-nyó- ${ }^{{ }^{a}}$ i-N-yogá

NEG-PS ${ }_{2}$-savoir-SBJV que $\mathrm{PS}_{2 \mathrm{sg}}$-boire-REL.VF $\mathrm{AUG}_{9}$ - $\mathrm{PN}_{9}$-bière

i-ó i-N-héke gir-a u-tyá

$\mathrm{PP}_{9}$-CONN $\mathrm{AUG}_{9}$ - $\mathrm{PN}_{9}$-bière.de.sorgho dire-VF $\mathrm{PS}_{2 \mathrm{sg}}$-comme.ça

u-ti N-a-meeng-a si i-N-héke

$\mathrm{PS}_{2 \mathrm{sg}}$-QUOT $\mathrm{PS}_{1 \mathrm{sg}}$-PH-penser-VF COP.NEG AUG $\mathrm{AU}_{9}-\mathrm{PN}_{9}$-bière.de.sorgho

Ils dirent à la reine-mère : « Tais-le, pour qu'on ne sache pas que tu bois de la bière de sorgho. Dis comme ça : Je pensais que ce n'était pas de la bière de sorgho. ".

(Imigani, Contes, 1940s)

Etant donné que le $\mathrm{PS}_{1 \text { sg }}$ a une vraie valeur référentielle en (42), tout comme la marque de temps -a- a un véritable sens grammatical, -meeng- peut être considéré comme un vrai radical verbal et non plus comme un verbe défectif. Non seulement on observe d'ailleurs une resémantisation de la marque de la personne en (42), mais aussi de ce radical verbal qui a développé un nouveau sens lexical. Celui-ci est proche du sens cognitif du verbe d'origine -meny- "savoir », qui fait aussi partie de l'exemple en (42), mais il est encore plus proche du sens " croire ; penser » du verbe polysémique -girillustré en (43). Les deux verbes, -gir- et -meeng- ont d'ailleurs en commun de pouvoir introduire une subordonnée sans la présence d'un complémenteur comme kó ou ngo. Tout comme le verbe de base -meny- " savoir », le nouveau verbe -meeng- « penser » n'accepte pas d'être suivi par ngo en kirundi dit commun. 
(43) Wagira ndatinya kubisubiramwo?

u-a-gir-a N-ra-tíny-a ku-bi-súbir-a-mwó

$\mathrm{PS}_{2 \mathrm{sg}}$-PH-penser-VF $\mathrm{PS}_{1 \mathrm{sg}}$-DJ-avoir.peur-VF $\mathrm{PN}_{15}-\mathrm{PO}_{8}$-retourner-VF-POSTF 18

Tu pensais que j'aurai peur de le répéter?

(Gikenye, Théâtre, 1970s)

Dans la région de Buhonga à l'Imbo central, l'évolution de -meeng- est encore plus avancée. Il s'y emploie systématiquement comme verbe lexical avec la possibilité du perfectif et d'emploi de personnes autres que la 1sg, contrairement aux faits observés en kirundi dit commun et dans les autres variantes, où le paradigme de la personne est limité à la 1sg et à la 2sg et ne se combine pas avec la désinence perfective. Par contre, le verbe y a aussi le sens de «croire, penser ». En plus, contrairement au kirundi dit commun, il peut être suivi du quotatif ngo introduisant une autre proposition dont la vérité n'est pas garantie. L'exemple fourni en (44) illustre ce particularisme régional.

\section{(44) Ntiyaguze amavúta kukó yameenze ngo turacáayafíse muu nzu.}

nti-a-a-gur-ye a-ma-vúta kukó a-a-meeng-ye

NEG-PS 3 sg $-\mathrm{PH}$-acheter-PFV $\quad \mathrm{AUG}_{6}-\mathrm{PN}_{6}$-huile parce.que $\mathrm{PS}_{3 \mathrm{sg}}$-PH-croire-PFV ngo tu-racáa-a-fít-ye $\mathrm{mu} \quad \mathrm{N}$-zu

QUOT PS ${ }_{1 \mathrm{pl}}$-PERST-PO ${ }_{6}$-avoir-PFV LOC $_{18} \quad \mathrm{PN}_{9}$-maison

Il n'a pas acheté de l'huile, parce qu'il a cru que nous en avions encore dans la maison.

(kirundi de Buhonga; élicitée)

Cet emploi verbal illustre une autre étape d'évolution aussi bien sur le plan de la forme qu'au niveau du sens, où -meeng- fonctionne comme un verbe à part entière et acquiert un sens nouveau, un sens cognitif qui ne s'est pas encore étendu à tous les usagers du kirundi. Cette étape est pertinente dans la description des mécanismes que subit umeenga/umeengo. Il s'agit d'une évolution par analogie, fondée sur la reproduction des règles ordinaires de la finale verbale.

L'émergence de ce nouveau radical verbal à partir de l'usage comparatif de l'adverbe umeengo/umeenga n'est pas tout à fait récente, car plusieurs de nos prédécesseurs en font mention, quoique de façon assez superficielle. Rodegem (1970 : 262) inclut dans son dictionnaire un lemme kumênga, qu'il traduit comme " croire, juger, se douter de ; penser, avoir l'impression ». Néanmoins, un locuteur du kirundi trouverait inusitée cette forme infinitive. Elle n'est pas non plus attestée dans notre corpus. Rodegem (1970 : 262) y évoque aussi la 
possibilité d'une forme perfective marquée par la finale -ye. Comme nous venons d'expliquer, ce n'est que dans le parler de la région occidentale de l'Imbo qu'on retrouve ce verbe avec cette finale. Dans cette région, les locuteurs tendent à l'utiliser comme verbe à part entière avec comme sens « croire ; penser ». Zorc \& Nibagwire (2007 : 292) identifient aussi « -méengo (alt. -méenga) » comme un verbe défectif signifiant «say that; suppose, believe; think, have an impression (that); seem (to be so) » et appartenant donc à la classe des verbes cognitifs : " a small set of verbs [...] that expresses some form of knowing (eg. supposing, perceiving, or being acquainted)».

\section{Conclusions}

Grâce à une étude approfondie des différentes formes et usages de umeengo/ umeenga à la fois en kirundi dit commun et dans plusieurs de ses variantes régionales ainsi que dans quelques langues voisines, nous sommes en mesure d'en déduire l'origine. Celle-ci ne peut se déduire directement d'aucune forme actuellement en usage dans le kirundi dit commun sur lequel notre corpus est basé. Son parcours implique des évolutions que l'on pourrait caractériser comme la grammaticalisation et la dégrammaticalisation.

Dans l'évolution de ces formes, il se passe une série de processus à la fois sur le plan formel et du point de vue sémantique dans l'émergence et l'emploi des formes umeengo et umeenga. Au stade préliminaire, une nouvelle unité se forme à travers la fusion de deux mots (umenya + ngo) et l'attrition phonologique sur le plan formel, et par l'érosion de la personne et du sens lexical du verbe sur le plan sémantique. Le déroulement parallèle et graduel des processus d'univerbation et d'érosion sémantique est bien typique de la grammaticalisation (Haspelmath 2004, Lehmann 2004, Norde 2009). Etant donné que l'adverbe umeengo/umeenga est un nouveau mot à part entière, sa formation peut également être considérée comme un cas de lexicalisation, un processus qui se distingue bien évidemment de la grammaticalisation, mais qui implique aussi souvent la fusion de mots et l'attrition phonologique. Il est bien connu qu'à la fois la grammaticalisation et la lexicalisation contribuent à la création d'adverbes et que les deux sont souvent difficiles à démêler dans ce processus (Ramat 2011 : 506-509). Par ailleurs, ce qui se passe dans la naissance de l'adverbe umeengo/umeenga va au-delà de la simple formation des mots. Il résulte de la fusion d'un verbe et d'un complémenteur. Heine et al. (1991 : 3) considèrent que la mutation de mots appartenant à une classe ouverte, comme celle des verbes, vers une catégorie fermée, comme celle des adverbes, relève de la 
grammaticalisation. En plus, son usage comme marque épistémique est bien grammatical, dans le sens où certains affixes verbaux comme la marque modale -oo- revêtent des sémantismes très proches. Considérons également le rôle de la subjectification que nous entendons ici comme un changement sémantique caractérisé par l'évolution de sémantismes plus objectifs relatifs au monde extralinguistique vers des sémantismes qui se basent plutôt sur l'attitude ou le point de vue subjectif du locuteur vis-à-vis de la proposition (Traugott 1989, p. 35). Cette évolution sémantique, qui est inhérente même à la formation des adverbes épistémiques, est connue pour être étroitement associée à la grammaticalisation dans le domaine de la modalité (Traugott 2010) et a également été observée en association avec d'autres marques modales en kirundi (Bostoen et al. 2012).

Si l'adverbe umeengo/umeenga est issu des processus de grammaticalisation et lexicalisation, il a engendré lui-même un nouveau verbe à travers une évolution que l'on pourrait caractériser comme la dégrammaticalisation telle que définie par Norde (2009). Il s'agit en même temps d'une délexicalisation dans le sens où l'adverbe perd son statut de mot unique et figé. Ses différents composants historiques qui avaient perdu leur statut morphologique dans le processus d'univerbation sont remorphologisés avec un sens qui est soit identique soit différent de leur fonction étymologique. Le résultat ne peut plus être considéré un véritable adverbe. Dans son usage comparatif, umeengo/ umeenga a développé la capacité d'intégrer de la morphologie flexionnelle typique du verbe, comme certaines marques de temps et d'aspect. Dans ce processus, le $\mathrm{PS}_{2 \mathrm{sg}}$ qui avait été désémantisé au cours du processus de formation de l'adverbe s'est resémantisé et a regagné son statut morphologique indiqué par le fait qu'il peut commuter avec le $\mathrm{PS}_{1 \text { sg }}$ dans le kirundi dit commun et avec tout autre PS dans la variété régionale occidentale de Buhonga. Dans cette dernière, même la voyelle finale a été (re)morphologisée dans le sens où elle peut commuter avec la désinence perfective -ye. Du point de vue de l'adverbe même, cette remorphologisation de ses différents composants historiques peut être considérée comme une délexicalisation. Toutefois, du point de vue des composants eux-mêmes, il s'agit plutôt d'une relexification. Ils acquièrent de nouveau leur statut de mot indépendant appartenant respectivement aux classes des verbes et des conjonctions. Le nouveau radical verbal -meeng- " penser, croire » qui en émerge peut être conçu, quant à lui, comme un cas de dégrammaticalisation. Tant la nouvelle forme que le nouveau sens lexical qui en résultent se distinguent de ceux du verbe étymologique -meny" savoir ». Ce processus, tout en étant l'inverse du premier, ne constitue donc pas un retour à la situation initiale. Il y a émergence d'un nouveau radical verbal -meeng- qui a un sens tout nouveau et la capacité à entrer en relation 
syntagmatique avec d'autres constituants du verbe comme la personne et les marques de temps et d'aspect. Dans le kirundi dit commun, ce verbe est encore défectif. Dans la variété de Buhonga, il est devenu un verbe lexical à proprement parler. Quoique difficile à prouver avec certitude, il semble que l'évolution de l'adverbe umeengo vers un nouveau verbe défectif en kirundi dit commun soit reflétée dans notre corpus comme cela est schématisé dans la Figure 2 présentée ci-dessus. Bien que la grammaticalisation de l'adverbe umeengo/umeenga soit considérablement plus ancienne que la période couverte par notre corpus, la réduction de moitié qu'a subie l'usage de umeengo depuis les années 1940s par rapport à l'usage constant de sa variante umeenga pourrait indiquer que la dégrammaticalisation de umeengo, c'est-à-dire le redéveloppement de propriétés verbales, se fait au détriment de son usage adverbial et donc de sa fréquence générale.

Le parcours de umeengo/umeenga peut être ainsi représenté à l'aide de la Figure 3, qui rend compte de la part des deux mouvements, à savoir la grammaticalisation et lexicalisation conjointes vers un adverbe dans les étapes 2 et 3 et la dégrammaticalisation et délexicalisation conjointes vers un nouveau verbe dans les étapes 4 et 5 . La variante qui apparaît par analogie à la troisième étape facilite les étapes ultérieures.

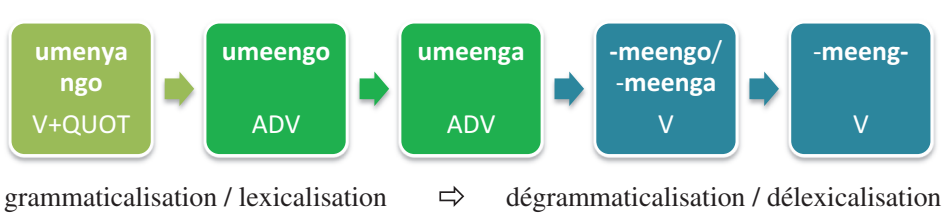

Figure 3: Formalisation des processus de grammaticalisation/dégrammaticalisation et de lexicalisation/délexicalisation de umeengo/umeenga.

\section{Abréviations}

$\begin{array}{ll}\text { APPL } & \text { applicatif } \\ \text { ASSOC } & \text { associatif } \\ \text { AUG } & \text { augment } \\ \text { CAUS } & \text { causatif } \\ \text { CJC } & \text { mode conjonctif } \\ \text { CONN } & \text { connectif } \\ \text { COP } & \text { copule } \\ \text { DEM } & \text { démonstratif, de degré x } \\ \text { DJ } & \text { disjoint } \\ \text { EV } & \text { éventuel }\end{array}$




$\begin{array}{ll}\text { FUT } & \text { futur } \\ \text { H } & \text { ton haut marqueur de mode } \\ \text { HYP } & \text { hypothétique } \\ \text { IMP } & \text { impératif } \\ \text { IMPO } & \text { impositif } \\ \text { INTENS } & \text { intensif } \\ \text { INTERR } & \text { interrogatif } \\ \text { IPFV } & \text { imperfectif } \\ \text { LOC } & \text { locatif } \\ \text { MOD } & \text { modalité } \\ \text { N } & \text { nasale homorganique } \\ \text { NEG } & \text { négatif } \\ \text { OBJ } & \text { objet } \\ \text { PASS } & \text { passif } \\ \text { PE } & \text { passé éloigné } \\ \text { PERST } & \text { persistif } \\ \text { PFV } & \text { perfectif } \\ \text { PH } & \text { passé hodiernal } \\ \text { pl } & \text { plural } \\ \text { PN } & \text { préfixe nominal } \\ \text { PO } & \text { préfixe objet } \\ \text { POSS } & \text { possessif } \\ \text { POT } & \text { potentiel } \\ \text { POSTF } & \text { postfinale } \\ \text { PP } & \text { préfixe pronominal } \\ \text { PRCS } & \text { précessif } \\ \text { PRES } & \text { présentatif } \\ \text { PS } & \text { préfixe sujet } \\ \text { QUOT } & \text { quotatif } \\ \text { REFL } & \text { réfléchi } \\ \text { REL } & \text { mode relatif } \\ \text { SBJV } & \text { subjonctif } \\ \text { Sg } & \text { singulier } \\ \text { SUBSEC } & \text { subsécutif } \\ \text { SUBST } & \text { substitutif } \\ \text { VF } & \text { voyelle finale } \\ \text { VOC } & \text { vocatif } \\ & \end{array}$

\section{Références}

Aikhenvald, Alexandra Y. 2004. Evidentiality. Oxford: Oxford University Press.

Bastin, Yvonne, André Coupez, Evariste Mumba \& Thilo C. Schadeberg (eds). 2002. Bantu Lexical Reconstructions 3 / Reconstructions lexicales bantoues 3. Tervuren : Royal Museum for Central Africa. Online at http://www.africamuseum.be/collections/browsecollections/ humansciences/blr. 
Bostoen, Koen, Ferdinand Mberamihigo \& Gilles-Maurice de Schryver. 2012. Grammaticalization and subjectification in the semantic domain of possibility in Kirundi. Africana Linguistica 18. 6-40.

Bostoen, Koen \& Ernest Nshemezimana. à paraître. The conjoint/disjoint alternation in Kirundi (JD62): A case for its abolition. In J. van der Wal \& L. M. Hyman (eds.), Berlin; New York: De Gruyter.

Charaudeau, Patrick. 1992. Grammaire du sens et de l'expression. Paris: Hachette.

Cornillie, Bert. 2009. Evidentiality and epistemic modality. On the close relationship between two different categories. Functions of Language 16. 44-62.

Coupez, André, Thomas Kamanzi, Simon Bizimana, Gaspard Sematama, Gaspard Rwabukumba, Charles Ntazinda \& collaborateurs. 2005. Dictionnaire rwanda-français et françaisrwanda / Inkoranya y'ikinyarwaanda mu kinyaarwaanda nó mu gifaraansá. Tervuren : Musée royal de l'Afrique centrale.

Cristini, Giovanni. 2001. Indīmbūro y’íkirǔndi. Nouvelle grammaire du kirundi. Bujumbura : Presses Lavigerie.

Devos, Maud \& Koen Bostoen. 2012. Bantu do/say polysemy and the origins of a quotative in Shangaci. Africana Linguistica 18. 97-132.

Dubois, Jean, Mathée Giacomo \& Louis Guespin (eds). 2007. Grand dictionnaire : linguistique et sciences du langage. Paris: Larousse.

Frey, Claude. 1996. Le français au Burundi. Lexicographie et culture. Vanves: EDICEF.

Haspelmath, Martin. 2004. On directionality in language change with particular reference to grammaticalization. In Olga Fischer, Muriel Norde \& Harry Perridon (eds.), Up and down the cline: the nature of grammaticalization (Typological Studies in Language 59), 17-44. Amsterdam; Philadelphia: John Benjamins.

Heine, Bernd, Ulrike Claudi \& Friederike Hünnemeyer. 1991. Grammaticalization : a Conceptual Framework. Chicago : University of Chicago Press.

Hopper, Paul J. \& Elizabeth C. Traugott. 2003. Grammaticalization (Cambridge Textbooks in Linguistics). Cambridge; New York: Cambridge University Press.

Hoye, Leo. 1997. Adverbs and Modality in English. London; New York: Longman.

Kagame, Alexis. 1969. Introduction aux grands genres lyriques de l'ancien Rwanda. Butare : Université nationale du Rwanda.

Kawalya, Deo, Koen Bostoen \& Gilles-Maurice de Schryver. 2014. Diachronic semantics of the modal verb -sóból- in Luganda : A corpus-driven approach. International Journal of Corpus Linguistics 19. 60-93.

Le Querler, Nicole. 1996. Typologie des modalités. Caen : Presses universitaires de Caen.

Le Roux, Jurie C. 2007. A Grammatical Analysis of the Tswana Adverbial (Thèse de doctorat). Pretoria: University of South Africa.

Lehmann, Christian. 2004. Theory and method in grammaticalization. Zeitschrift für Germanistische Linguistik 32(2). 152-87.

Mberamihigo, Ferdinand. 2014. L'expression de la modalité en kirundi. Exploitation d'un corpus électronique (Thèse de doctorat). Bruxelles/Gand : Université libre de Bruxelles (ULB), Université de Gand (UGent).

Meeussen, Achiel E. 1959. Essai de grammaire rundi. Tervuren : Musée royal du Congo belge.

Mould, Martin. 1977. On the productivity of derivational morphology and lexical representations: Manner adverbs in Luganda. In M. Mould \& T. J. Hinnebusch (eds.), Proceedings of the 8th Conference on African Linguistics, 175-83. Los Angeles: African Studies Center \& Dept. of Linguistics, University of California at Los Angeles (UCLA). 
Narrog, Heiko. 2010. (Inter)subjectification in the domain of modality and mood - Concepts and cross-linguistic realities. In K. Davidse, L. Vandelanotte \& H. Cuyckens (eds.), Subjectification, Intersubjectification, Grammaticalization (Topics in English Linguistics), 385-430. Berlin; New York : Mouton de Gruyter.

Norde, Muriel. 2009. Degrammaticalization (Oxford Linguistics). Oxford; New York: Oxford University Press.

Ntahokaja, Jean-Baptiste. 1994. Grammaire structurale du kirundi. Bujumbura : Université du Burundi.

Nuyts, Jan. 2005. The modal confusion: on terminology and concepts behind it. In Alex Klinge \& Henrik H. Müller (eds.), Modality: Studies in Form and Function, 5-38. London: Equinox.

Nuyts, Jan. 2006. Modality: Overview and linguistic issues. In William Frawley, Erin Eschenroeder, Sarah Mills \& Thao Nguyen (eds.), The Expression of Modality (The expression of cognitive categories 1), 1-26. Berlin; New York: Mouton de Gruyter.

Plungian, Vladimir A. 2001. The place of evidentiality within the universal grammar space. Journal of Pragmatics 33(3).349-57.

Ramat, Paolo. 2011. Adverbial grammaticalization. In Heiko Narrog \& Bernd Heine (eds.), The Oxford Handbook of Grammaticalization, 502-510. Oxford: Oxford University Press.

Rodegem, Firmin M. 1967. Précis de grammaire rundi. Brussels; Ghent : Story-Scientia.

Rodegem, Firmin M. 1970. Dictionnaire rundi-français. Tervuren : Musée royal de l'Afrique centrale.

Segopolo, B. 0. 1992. The adverb in Tswana. In A. J. Lopes (ed.), Proceedings of the 3rd LASU conference/workshop, Maputo '91, 229-52. Maputo: Univ. Eduardo Mondlane Press; Linguistics Association for SADC Universities (LASU).

Traugott, Elizabeth C. 1989. On the rise of epistemic meanings in English : An example of subjectification in semantic change. Language 65. 31-55.

Traugott, Elizabeth C. 2010. '(Inter)subjectivity and (inter)subjectification: A reassessment'. In Kristin Davidse, Lieven Vandelanotte \& Hubert Cuyckens (eds.), Subjectification, Intersubjectification, Grammaticalization (Topics in English Linguistics), 29-71. Berlin; New York: Mouton de Gruyter.

van der Auwera, Johan \& Vladimir A. Plungian. 1998. Modality's semantic map. Linguistic Typology 2. 79-124.

Van Otterloo, Roger. 2011. The Kifuliiru Language. Volume 2. A Descriptive Grammar. Dallas: SIL International.

WordSmith Tools. Available online at: http://www.lexically.net/wordsmith/.

Zorc, R. David \& Louise Nibagwire. 2007. Kinyarwanda and Kirundi Comparative Grammar. Hyattsville, MD: Dunwoody Press. 